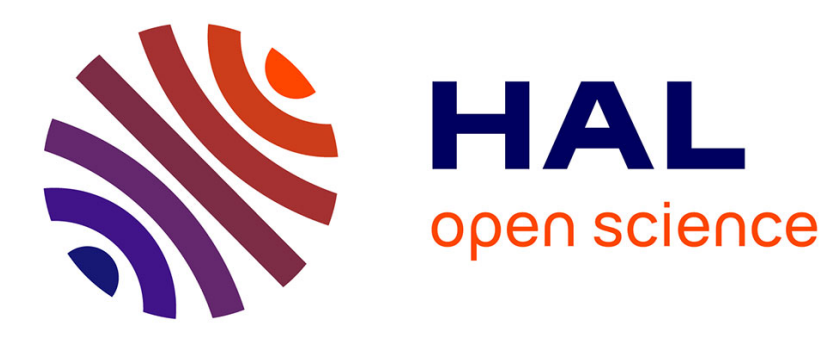

\title{
Ethylene signaling modulates tomato pollen tube growth, through modifications of cell wall remodeling and calcium gradient
}

Rasha Althiab-almasaud, Yi Chen, Elie Maza, Anis Djari, Pierre Frasse, Jean-claude Mollet, Christian Mazars, Elisabeth Jamet, Christian Chervin

\section{To cite this version:}

Rasha Althiab-almasaud, Yi Chen, Elie Maza, Anis Djari, Pierre Frasse, et al.. Ethylene signaling modulates tomato pollen tube growth, through modifications of cell wall remodeling and calcium gradient. Plant Journal, 2021, 107 (3), pp.893-908. 10.1111/tpj.15353 . hal-03246733

\section{HAL Id: hal-03246733}

https://hal-normandie-univ.archives-ouvertes.fr/hal-03246733

Submitted on 3 Jun 2021

HAL is a multi-disciplinary open access archive for the deposit and dissemination of scientific research documents, whether they are published or not. The documents may come from teaching and research institutions in France or abroad, or from public or private research centers.
L'archive ouverte pluridisciplinaire HAL, est destinée au dépôt et à la diffusion de documents scientifiques de niveau recherche, publiés ou non, émanant des établissements d'enseignement et de recherche français ou étrangers, des laboratoires publics ou privés. 


\section{Ethylene signaling modulates tomato pollen tube growth, through modifications of cell wall remodeling and calcium gradient}

Rasha Althiab-Almasaud ${ }^{1 *}$, Yi Chen ${ }^{1,2}$, Elie Maza ${ }^{1}$, Anis Djari ${ }^{1}$, Pierre Frasse ${ }^{1}$, Jean-Claude Mollet $^{3}$, Christian Mazars ${ }^{4}$, Elisabeth Jamet ${ }^{4}$, Christian Chervin ${ }^{1 *}$

1 Laboratoire de Génomique et Biotechnologie des Fruits, Université de Toulouse, Toulouse INPENSAT, INRAE, Auzeville-Tolosane, France

${ }^{2}$ College of Food and Pharmaceutical Sciences, Ningbo University, Ningbo, China

3 Normandie Univ, UniRouen, Laboratoire Glyco-MEV, SFR NORVEGE, Innovation Chimie Carnot, Rouen, France

${ }^{4}$ Laboratoire de Recherche en Sciences Végétales, Université de Toulouse, CNRS, UPS, Auzeville-Tolosane, France

\section{RUNNING TITLE}

Ethylene and pollen tube growth

Keywords: calcium signaling, ethylene perception, pectin methylesterase, pollen tube growth, Solanaceae, tomato

*Correspondence: christian.chervin@toulouse-inp.fr, ENSAT, BP 32607, 31326 CastanetTolosan, France

\section{SUMMARY}

Ethylene modulates plant developmental processes including flower development. Previous studies have suggested ethylene participation in pollen tube (PT) elongation, and both ethylene production and perception seem critical at fertilization time. The full gene set regulated by ethylene during PT growth is unknown. To study this, we used various EThylene Receptors (ETRs) tomato mutants: etr3-ko, a loss-of-function (LOF) mutant; and NR (Never Ripe), a gain of function (GOF) mutant. The etr3-ko PTs grew faster than its wild type, WT. Oppositely, NR PT elongation was slower than its wild type, and PTs displayed larger diameters. ETR mutations created feedbacks on ethylene production. Furthermore, the ethylene treatment of germinating pollen grains increased PT length in etr-ko mutants and WT, but not in NR. Treatments with the

This article has been accepted for publication and undergone full peer review but has not been through the copyediting, typesetting, pagination and proofreading process, which may lead to differences between this version and the Version of Record. Please cite this article as doi: $\underline{10.1111 / \text { tpj.15353 }}$

This article is protected by copyright. All rights reserved 
ethylene perception inhibitor, 1-MCP, decreased PT length in etr-ko mutants and wild types, but had no effect on NR. This confirmed that ethylene regulates PT growth. The comparison of PT transcriptomes in LOF and GOF mutants, etr3-ko and NR, both mutated on the ETR3 gene, revealed that ethylene perception has major impacts on cell wall- and calcium-related genes as confirmed by microscopic observations showing a modified distribution of the methylesterified homogalacturonan pectic motif and of calcium load. Our results establish links between PT growth, ethylene, calcium and cell wall metabolisms, and also constitute a transcriptomic resource.

\section{INTRODUCTION}

The human population growth and increasing food demand make food security a priority for the future (Gustavsson et al., 2011). Therefore, research on plant reproduction is critical for increasing crop productivity and fruit/seed yields. Sexual plant reproduction starts with pollen grain landing onto the stigma where it hydrates and starts to germinate; then, the pollen tube (PT), carrying the two sperm cells, grows rapidly through the style and the ovary (Figure S1), to reach the ovule where the fertilization takes place (An et al., 2020). PT elongation is essential for fertilization (Michard et al., 2017). Many studies suggested that ethylene participates in this process (An et al., 2020). For example, a few hours after the penetration of PTs in the tomato stigma, a burst of ethylene was detected in the pistil (Llop-Tous et al., 2000). Ethylene signaling starts with the binding of ethylene to specific receptors (Chang, 2016, Binder, 2020). In tomato, there are seven EThylene Receptors (ETRs), SIETR1-7, which are located in the endoplasmic reticulum, and their amount varies over plant development (Chen et al., 2020a). ETRs are negative regulators of ethylene responses. In absence of ethylene, they maintain the downstream signaling partners in a "OFF" status, but in presence of ethylene their phosphorylation level changes and this turns "ON" the signaling partners (Bisson \& Groth, 2015; Chang, 2016; Binder, 2020).

Some studies have highlighted the critical role of ethylene perception in PT elongation. For example, in Petunia inflata, ethylene perception was shown to be correlated with PT elongation (Holden et al., 2003). Indeed, the treatment with 1-MethylCycloPropene (1-MCP), an ethylene perception inhibitor, led to a strong inhibition of PT growth during the first six hours after pollination. Besides, Jia et al. (2018) have observed that ethylene promotes PT growth by interacting with cyclic Guanosine MonoPhosphate (cGMP) in Arabidopsis thaliana, thus leading to 
an increased number of actin filaments in PTs, which are important for their polarized growth. Moreover, in an ethylene-insensitive mutant (etr1-1), PT growth was reduced (Jia et al., 2018).

During PT growth, a number of cell wall components like pectins, cellulose, hemicelluloses and callose are required to build up the cell wall architecture (Mollet et al., 2013). Pectins constitute a major component of the PT apical region, and the pectin methylesterase (PME) activity is critical for PT growth (Bosch et al., 2005). During fruit ripening, ethylene was shown to modulate the expression of genes encoding cell-wall modifying enzymes such as PMEs and polygalacturonases (PGs) (Brummel, 2006). PGs are also known to be critical for PT growth (Dearnaley and Daggard, 2001). But a clear link between ethylene and cell wall remodelling during PT growth is yet to be demonstrated.

PT elongation, morphology, and orientation have been shown to be highly dependent on ions choreography. Namely, the combination of potassium, chloride, calcium and proton gradients play crucial roles in the development of pollen grains (Michard et al., 2017). Tip-focused $\mathrm{Ca}^{2+}$ gradients generated by the interplay of $\mathrm{Ca}^{2+}$ pumps and channels are observed during PT elongation and are correlated with pulses in growth rate (Zheng et al., 2019). The link between $\mathrm{Ca}^{2+}$ and ethylene has been reported, in other organs than flowers. It was found that $\mathrm{Ca}^{2+}$ is required for ethylene biosynthesis (Jung., 2000; Petruzzelli et al., 2003; Li., 2018). Moreover, it was shown that ethylene could enhance the endogenous $\mathrm{Ca}^{2+}$ concentration in tobacco cell suspension cultures by activating a plasma membrane $\mathrm{Ca}^{2+}$-permeable channel (Zhao et al., 2007). A recent study has shown that exogenous application of $\mathrm{Ca}^{2+}$ to root tissues was able to down-regulate the expression of ETR genes, and up-regulate gene expression of downstream elements of the ethylene signaling pathway (Yu et al., 2019).

In the present study, we aimed at investigating the role of ETRs during PT growth in tomato. After identifying the main ETRs expressed during PT growth, we studied the effects of mutations affecting ETRs with opposite effects, a loss-of-function (LOF) mutation in the etr3 mutant, and a gain-of-function (GOF) mutant carrying a constitutive functional ETR (NR, for NEVER RIPE). A RNA-seq comparative study between etr3-ko, NR and their respective wild-types, led us to focus on $\mathrm{Ca}^{2+}$ signaling- and cell wall-related genes, and on pectin remodeling to understand the observed phenotypes. In this study, we show links between metabolisms related to ethylene, calcium and cell wall during PT growth.

\section{RESULTS}

This article is protected by copyright. All rights reserved 


\section{Expression of ETRs in germinating pollen grains}

In WT PTs, we observed that ETR3, ETR4, and ETR5 were the ETR genes with the highest expression levels $4 \mathrm{~h}$ after pollen imbibition, without significant differences between them (Figure 1A). ETR1, 2 and 7 were expressed at significantly lower levels, and ETR6 transcripts were below the detection threshold. A preliminary experiment showed that $4 \mathrm{~h}$ after imbibition was the optimum time at which we were able to collect a sufficient amount of raw material and extract total RNA of good quality, with PTs being still in a linear phase of growth (Figure S2). In the following parts, we will focus on etr3, since we have LOF and GOF mutants for this gene, in the search for changes in gene expression between the two mutant types, to reveal the metabolisms driving PT growth under ETR control. Moreover, ETR3 has been shown to be involved in PT growth (Jegadeesan et al., 2018).

\section{ETRs modulate pollen tube growth, but not ethylene production by pollen tubes}

To test whether ETRs modulate PT growth, we used two ETR3 mutants: the etr3-ko line, a LOF mutant, and the NR line, a GOF mutant of etr3, and their corresponding wild types, i.e. WT and WTB, respectively (see Experimental Procedures for details). After $4 \mathrm{~h}$ of imbibition, PT growth was increased in etr3-ko, whereas PT growth was reduced in NR (Figure 1B). We observed a weak ethylene production by PTs, but without any significant difference between the tomato lines (Figure 1C). A similar stimulation of PT growth was also observed in another LOF affecting ETR4, etr4-ko (Figure S2A detailing growth kinetics), and in addition, we observed that the PTs of the etr3-ko/etr4-ko double mutant were growing faster than those of WT, but not significantly faster than those of etr3-ko or etr4-ko single mutants (Figure S2B).

\section{Ethylene stimulates pollen tube growth, whereas 1-MCP inhibits it}

We decided to check the impact of adding an ETR effector, the ethylene itself, or adding an ETR inhibitor, 1-methylcyclopropene (1-MCP) in the headspace above germinating pollen grains. In etr3-ko, and in both WT lines, the ethylene treatment stimulates PT elongation (Figure 1B). This was not the case in NR, which is ethylene-insensitive. On the contrary, the addition of 1-MCP limited PT growth in etr3-ko (Figure 1B), but no significant change was observed in both WT and $N R$ lines. After showing that functional ETRs limit PT growth, an effect reversed by adding ethylene, we performed an RNA-seq experiment to look at changes in gene expression possibly driven by ETR signaling and impacting PT growth.

\section{Transcriptome profiling of etr3-ko and NR pollen tubes}


We compared the PT mRNAs of two etr3 mutant lines, etr3-ko (ETR3 LOF) and NR (ETR3 GOF), and their respective WTs. Total RNA was extracted from PTs collected $4 \mathrm{~h}$ after pollen grain imbibition. The overall quality of sequencing data was very good with a mean read quality of 35 (Illumina 1.9 encoding) (Figure S3A). A cleaning step was performed as $40.8 \%$ of the reads still had sequencing adaptors. After this step, more than $94 \%$ of the reads were mapped without ambiguity and less than $4 \%$ were mapped at more than one location (Figure S3B). This showed a very good specificity of sequences confirmed by RNA-seq quantification. As shown in Figure $\mathrm{S} 3 \mathrm{C}$, more than $90 \%$ of sequenced reads were assigned to genes after the mapping process, making the analysis even more robust.

A principal component analysis (PCA), carried out on normalized RNA-seq counts, showed that the four biological replicates were well-clustered in the four conditions WT, WTB, etr3-ko, and NR (Figure 2A). This showed that all samples were of good quality, and that the biological variability was smaller than differences between the four conditions of interest. Moreover, the PCA revealed that the horizontal axis (first axis of the PCA), carrying $42 \%$ of the variability, discriminated the NR mutant from the three other conditions, which meant that $N R$ was the condition creating the biggest differences. On the second axis carrying $26 \%$ of the variability, this PCA also underlined that both WT lines were close to each other. It could also be seen that etr3-ko was closer to WTs than NR.

There were 2452 Differentially Expressed Genes (DEGs) in NR vs WTB and 551 DEGs in etr3-ko vs WT (Figure 2B): 1131 were up-regulated genes in NR vs WTB, and 253 in etr3-ko vs WT, whereas the numbers of down-regulated genes were 1321 and 298 , respectively. To validate the quality of the RNA-seq data by RT-qPCR analyses, we chose 15 genes, encoding proteins possibly impacting PT growth, and ranging from low to high expression levels in the RNA-seq experiment (Table S2). The chosen genes were associated to functions about ethylene perception or synthesis, or related to cell wall metabolism or calcium signaling. The data were generated with the same RNA samples as for the RNA-seq analysis. The values obtained from the four biological replicates were averaged, thus the correlation calculation between RNA-seq and RT-qPCR values was performed over 60 data points. The results showed a Pearson correlation coefficient of 0.864 , a $P$ value of $6.710^{-19}$ and a power of 1 (Table S2). These were good indicators of transcriptomic data quality. Because previous studies showed that tomato ETRs expression is altered in ETR mutants (Tieman et al., 2000, Chen et al., 2019), we checked if this was the case for the seven ETRs in PTs. The expression level of three ETRs was altered in the mutants: ETR4 and ETR5 were up-regulated in NR, whereas ETR3 was down-regulated in 
etr3-ko (Figure 2C). These results were confirmed by RT-qPCR (Table S2). The expression of the other genes, tested in the qPCR check, did not reveal any trend or will be described below.

The full list of DEGs, genes with a $P<0.05$, is available in Table S3. In the following part of the study, we focused on cell wall modification and calcium signaling based on the observation of DEG families (Figure 2D). This revealed that cell wall-related genes were up-regulated in etr3-ko vs WT and down-regulated in NR vs WTB, whereas calcium signaling-related genes were strongly down-regulated in NR vs WTB. The key genes attracting our attention, with opposite differences between GOF and LOF mutants were: pectin methylesterases (PMEs) involved in cell wall remodeling, and calcium-dependent protein kinases (CPKs), calcium-pumps (ACAs), and calcium channels (CNGCs) for calcium signaling. They will be detailed below.

\section{Transcriptomic analysis of the cell wall-related genes in etr3-ko and $N R$ pollen tubes}

In both comparisons (NR vs WTB and etr3-ko vs WT), around 10\% of genes showing modified transcript levels encode proteins involved in cell wall protein or polysaccharide biosynthesis, in signaling at the plasma membrane interface or in cell wall metabolism, e.g. cell wall polysaccharides modifications (Table S4). Among these genes, there were 192 DEGs in NR vs WTB, and 59 in etr3-ko vs WT. The encoded cell wall-related proteins belong to five main functional classes: proteins acting on cell wall polysaccharides (29\% in NR and in etr3-ko), proteases (11 vs 16\%), proteins possibly related to lipid metabolism (9 vs 11\%), proteins with interaction domains with cell wall polysaccharides or proteins (5 vs 10\%) and proteins of yet unknown function (33\% vs 20\%). Only a few genes encoding oxido-reductases, mostly multicopper oxidases showed modified transcripts levels (Sedbrook et al., 2002). Among the proteins of yet unknown function, six DEGs in NR encoded proteins with six conserved cysteines exhibiting the same spacing as thionins (Silverstein et al., 2007).

A main feature regarding the cell wall-related genes was that $17 \%$ and $28 \%$ of them encoded proteins possibly involved in the pectin metabolism in NR vs WTB and in etr3-ko vs WT, respectively (Figure 3 and Table S4). These proteins belong to several families: polygalacturonases (PGs), of the glycosyl hydrolase family $28(\mathrm{GH} 28)$, pectin methylesterases (PMEs), of the carbohydrate esterase family 8 (CE8), pectate lyases (PLs) of the PL1 family, PME inhibitors (PMEls), trichome birefringence-like (TBLs) protein family and Pro-rich, Arabinogalactan conserved Cysteines (PAC) proteins (Hocq et al., 2017, Stranne et al., 2018, Nguyen-Kim et al., 2020). The number of DEGs encoding PGs and PMEs was higher in NR vs WTB than in etr3-ko vs WT. In NR, four PG genes had increased transcript levels, whereas four 
had decreased levels. Regarding the PME genes, three genes among the most highly expressed had slightly increased transcript levels, whereas seven PME genes were down-regulated (Table S4). In etr3-ko, four PME genes had higher transcript levels than in WT, among which two of the most highly expressed (Table S4). The PL genes showed the same kind of regulation in NR and etr3, with decreased transcript levels for four genes in NR and two in etr3-ko, and increased transcript levels for one gene in each case. In etr3-ko, two of the most highly expressed genes, Solyc01g068120 and Solyc06g084620, encode PMEs and showed increased transcript levels compared to WT. The transcript level of two PMEI genes was decreased in NR whereas the situation was more contrasted in etr3-ko with one gene having more transcripts and another one less. TBL genes also exhibited a complex situation with a reduced transcript levels for one gene in NR and an increased transcript levels for two genes in NR or of one gene in etr3-ko. This could be due to the fact that the encoded enzymes could have different types of substrates, either hemicelluloses or pectins. Finally, the transcript levels of two genes encoding PAC proteins showed opposite variations in NR, whereas one of them had less transcripts in etr3-ko.

Altogether, a significant proportion of the cell wall-related DEGs encoded proteins (i) exhibiting enzymatic activities (PG, PME, PL, PAE, and possibly TBL) directed against pectin polymers such as homogalacturonans (HGs) or rhamnogalacturonan I (RG-I) (Hocq et al., 2017, Stranne et al., 2018), (ii) regulating the enzymatic activity of PME (PMEI) (Giovane et al., 2004) or (iii) able to interact with RG-I (Hijazi et al., 2014).

Moreover, we observed that exogenous ethylene application stimulated the expression of three genes encoding PMEs (Solyc01g057220, Solyc05g052120 and Solyc12g099410) previously identified in the RNA-seq data set, and the application of 1-MCP slightly lowered their expression (Figure S4A). This further indicates that the expression of these genes is modulated by ethylene signaling.

\section{Transcriptomic analyses of calcium signaling-related genes in NR and etr3-ko pollen tubes}

The expression of $47 \mathrm{Ca}^{2+}$ signaling-related genes was significantly altered in NR and etr3-ko (Figure 4). There were 34 DEGs (26 down- and 8 up-regulated) in NR, and 13 DEGs (6 downand 7 up-regulated) in etr3-ko PTs. According to homologies with TAIR 11 annotation (Table S5), they belong to classes of $\mathrm{Ca}^{2+}$ pumps, $\mathrm{Ca}^{2+}$ channels, and $\mathrm{Ca}^{2+}$ sensors such as calmodulinbinding proteins (CaMBPs), calmodulin-like proteins (CMLs) or $\mathrm{Ca}^{2+}$ effectors including $\mathrm{Ca}^{2+}$ dependent protein kinases (CPKs) and CBL-interacting protein kinases (CIPKs). The DEGs 
coding for some proteins of similar function, were up-regulated in NR and down-regulated in etr3ko, or inversely. This could be related to inverse PT growth response in these mutants. Among these, CPKs were down-regulated in NR and up-regulated in etr3-ko. In contrast, CIPKs were down-regulated in $N R$, but not significantly affected in etr3-ko. Globally in $N R$, there were more down-regulated $C M L$ and CaM genes than in etr3-ko. Some of these down-regulated genes exhibited a high number of RNA copies, like Solyc06g068960, Solyc05g050750 and Solyc02g067220, when looking at the 'base mean' (Table S5), thus even DEGs with a small log2 fold change may have an impact on cell functions.

Additional results showed that exogenous ethylene application stimulated the expression of three $\mathrm{Ca}^{2+}$ signaling-related genes previously identified in the RNA-seq data set (Solyc01g010020, and Solyc02g063340, encoding calmodulins, and Solyc03g123890 encoding a $\mathrm{Ca}^{2+}$-transporting ATPase 1), and that the application of 1-MCP inhibited their expression (Figure S4B). This further shows that expression of these calcium signaling-related genes is modulated by ethylene signaling.

\section{Immuno-localization of homogalacturonans in etr3-ko and NR pollen tubes}

To check possible modifications of HGs in etr3-ko and NR PTs, we assessed the distribution of weakly and highly methylesterified HGs using LM19 and LM20, respectively. Labeling with LM20 revealed that the highly methylesterified HGs was, as expected, mainly located at the tip of the WT, WTB and etr3-ko PTs (Figure 5A). However, an unusual ring-like labeling pattern was observed along the NR PT cell wall (Figure 5A). Fluorescence intensity was measured in PT tips, using the same delimited area. The LM20 fluorescence intensity in etr3-ko PT apex slightly decreased compared to the WT, without being significant, while in NR PT tip, the intensity was significantly higher than in WTB and other lines (Figure 5B). Finally, the diameters of PTs grown in liquid and solid growth medium (GM) were measured in the tip region (Figure $5 \mathrm{C}-\mathrm{D}$ ). No difference was observed in solid GM (Figure 5D), whereas in liquid GM, the diameters of etr3-ko PTs were smaller than in WT, while NR PT diameters were larger than in WTB (Figure 5 C-D).

The immuno-localization with LM19 of epitopes associated with weakly methylesterified HGs gave no significant difference (Figure S5A and B): $50 \%$ and $57 \%$ of the PT tips of WT and WTB were labeled, respectively. In NR, $39 \%$ of the PT tips were labeled, vs $67 \%$ in etr3-ko.

More LM19 labeling means an increase in cell wall stiffness (Parre and Geitmann, 2005), but no conclusion can be taken here. In addition to immuno-localization, the total PME activity was assayed on whole protein extracts of PTs, but no significant difference was observed, neither 
looking at activity per fresh mass (Figure S5C), nor looking at specific activity per $\mu \mathrm{g}$ proteins (Figure S5D).

\section{Calcium load in pollen tube tips is modulated by ETRs}

To verify the ethylene signaling ability to change $\mathrm{Ca}^{2+}$ ion dynamics in PT apex, PTs were loaded with fluo-4/AM to highlight $\mathrm{Ca}^{2+}$ gradient from the tip to the base (Figure 6). Calcium loading was observed in WT, WTB, NR and etr3-ko PT tips (Figure 6: panels A-D) and the zone over which fluorescence intensity was quantified is delimited by a yellow dashed line (see Methods for timing details). The fluorescence intensity in the apex region was higher in etr3-ko compared with WT, while it was lower in NR compared with WTB (Figure 6E), indicating that ethylene signaling modulates the $\mathrm{Ca}^{2+}$ accumulation in the PT apex. This corroborates RNA-seq results, as ethylene signaling modulates the expression of several genes encoding pumps and channels involved in $\mathrm{Ca}^{2+}$ homeostasis, or sensors (CaMs, CMLs) and effectors (CDPKs, CIPKs) involved in $\mathrm{Ca}^{2+}$ decoding. In addition to microscopic imaging, we observed the effects of lanthanum, an inhibitor of calcium transport (Qu et al., 2016) on the PT growth of the different lines. Figure S6 shows that the application of ethylene increased $\mathrm{Ca}^{2+}$ accumulation in the WT PTs and that application of 1MCP decreased it. We also observed that the growth of all PTs was stopped after $2 \mathrm{~h}$ of lanthanum $\left(\mathrm{La}^{3+}\right)$ treatment, regardless of the tomato line and that the $\mathrm{Ca}^{2+}$ accumulation in $\mathrm{PT}$ decreased within 30 min following the $\mathrm{La}^{3+}$ treatment.

\section{DISCUSSION}

\section{Ethylene signaling modulates pollen tube growth}

A few previous studies have suggested a role for ethylene in PT growth (Holden et al., 2003; Jia et al., 2018), but the expression of ETRs in PTs has been rarely analysed. We observed that three genes, ETR3, ETR4 and ETR5, are expressed in tomato growing PTs, confirming recent observations showing expression of the same three ETRs in tomato flower tissues (Jegadeesan et al., 2018).

Using various etr mutants, we observed that LOF etr3-ko and etr4-ko led to longer PTs, and GOF $N R$, led to shorter PTs. This latter result confirms recent observations of shorter PTs in the Arabidopsis etr1-1 GOF mutant (Jia et al., 2018). The ethylene being produced without significant difference between all lines, this suggests that PT growth depends on signaling arising from ETRs. Then, PT growth enhancement observed in WT and WTB after adding ethylene suggests that ethylene releases the blocking effect imposed by ETRs, while the dominant NR mutation imposes a strong inhibition that ethylene cannot release at the chosen dose. Additionally, our 
results showing a significant increase in PT length upon ethylene treatment of the etr3-ko line, suggest that, as expected, knocking-out one ETR renders PTs more sensitive to ethylene signaling. PT growth stimulation in LOF mutants was modulated with a low dose of 1-MCP, and a significant decrease in PT length was observed in etr3-ko, whereas no significant decrease in PT length was observed in WT and WTB. Here, we confirm results regarding the effects of 1-MCP on PT growth in P. inflata (Holden et al., 2003), but unlike Arabidopsis (Jia et al., 2018), tomato PT growth does not appear to be governed by ETR1 alone. Altogether, it seems that ethylene signaling partially governs PT elongation. Indeed, blocking ethylene action by 1-MCP led to minimal PT growth, whatever the ETR alteration.

Before these experiments with LOF and GOF mutants, we ran preliminary trials with WT at various doses of ethylene and 1-MCP to determine optimal concentrations (Figure S7). The best stimulation of PT growth by ethylene was observed at 100 ppm, but adding ethylene up to 1000 ppm led to a decreasing effect on PT growth, may be due to slight toxicity. For 1-MCP, the dose chosen for all trials was $2 \mathrm{ppm}$, as the PT growth decrease seems to saturate beyond $4 \mathrm{ppm}$. It can be postulated that the remaining PT growth is independent of ethylene signaling.

\section{Transcriptome profiling of $N R$ and etr3-ko PTs revealed that the GOF mutation has a stronger impact than the LOF mutation}

Then we detected gene expression changes in ETR3 mutants using a RNA-seq survey. We showed that the number of DEGs between NR and WTB was higher than between etr3-ko and WT. These observations are logical considering that in NR, the ethylene-insensitive ETR3, dominates the signaling of the surrounding ETRs and blocks ethylene signaling (Chen et al., 2019), while the etr3-ko mutation impairs only one out of the three mostly expressed ETRs in PTs.

In addition, the expression of ETR3 was repressed in etr3-ko, without any consequence as a functional ETR3 protein is not produced. This negative feedback on other ETRs is not similar to recent observations in etr7-ko fruit tissues in which the expression of ETR3, ETR4, ETR5 and ETR6 was increased at the inception of ripening (Chen et al., 2020a). Thus, it is possible that the regulation of ETR expression is tissue-dependent. It is also possible that ETR3 and ETR7 do not exert similar feedback on ETR expression. In NR, the ETR4 and ETR5 transcripts are upregulated. The related proteins would increase the potential blockage of PT growth.

This article is protected by copyright. All rights reserved 


\section{Cell wall remodeling is a main output of ethylene signaling in pollen tubes: A focus on pectins}

The transcriptomics study revealed the importance of the changes observed in the expression levels of cell wall-related genes in both NR and etr3-ko PTs. The expression of genes encoding proteins involved in HG remodeling was the most impacted, as expected since HGs are abundant pectin components of PT cell walls (Dehors et al., 2019). Consequently, two kinds of enzymes could be modulated: HG-modifying enzymes demethylating or deacetylating HGs, such as PMEs and PAEs; or enzymes degrading HGs, thus producing oligogalacturonides, such as PGs or PLs (Hocq et al., 2017). In NR PTs, seven PME-, four PG-, and four PL-encoding genes were downregulated. This observation is consistent with PT length reduction. Indeed, PT elongation requires cell wall flexibility brought in part by the active remodeling of HGs at the PT tip, such as demethylation and reduction of HG chain length. When these modifications do not happen, the cell wall remains stiff, thus inhibiting PT elongation. Conversely, only a few genes encoding pectin-related genes were DEGs in etr3-ko PTs. Four PMEs were only slightly up-regulated, suggesting a little effect on HG methylesterification, but sufficient to explain the fastest growth compared to the other lines. Only one of these four PMEs was clearly down-regulated in NR, Solyc05g054360. Its level of expression went down from a log2 of 0.74 in etr3-ko to 0.1 in NR. Thus, the massive decrease in PME expression levels in NR was related to other genes.

Besides, transcript levels of TBL-encoding genes were strongly modified in NR and in etr3-ko. The precise role of the Arabidopsis 46 TBLs is unknown. However, they were grouped in five clusters, and clusters IV and V are linked to O-acetylation of hemicelluloses, like xyloglucan, mannan and xylan, whereas the other clusters gather TBLs possibly involved in O-acetylation of pectins (Stranne et al., 2018). The two TBL genes up-regulated in NR are homologous to Arabidopsis TBLs of cluster IV and V (Solyc07g053330, 2-fold; Solyc10g009590, 52-fold), suggesting a re-organization of the cell wall to cope with changes occurring at the pectin level. The third TBL gene with modified level of transcripts is down-regulated (about 2-fold) and is homologous to Arabidopsis TBL genes of cluster II among which the TBL44/PMR5 gene was shown to play a role in pectin esterification (Vogel et al., 2004). The expression of the Solyc07g053330 gene, coding for a putative cell wall polysaccharide O-acetyltransferase, was lower in etr3-ko than in NR, also suggesting modifications of hemicelluloses.

Since pectins are critical components for cell wall changes at the PT apex (Mollet et al, 2013), mainly by affecting cell wall rheological properties (Parre and Geitmann, 2005), and since regulation of genes involved in HG remodeling was affected in NR and etr3-ko, our study focused 
on HG distribution in PT cell walls. In WT, the weakly methylesterified HGs are located in the PT shank where they can be cross-linked by $\mathrm{Ca}^{2+}$ ions, promoting cell wall stiffness and providing mechanical support for the tip-polarized elongation (Dardelle et al., 2010; Chebli et al., 2012; Mollet et al., 2013; Dehors et al., 2019). In contrast, the highly methylesterified HGs are mainly located at the PT apical region providing sufficient flexibility required for the turgor-driven PT elongation (Parre \& Geitmann, 2005; Vogler et al., 2013; Mollet et al., 2013; Hepler et al., 2013)

The surprising LM20 labeling has revealed rings of highly methylesterified HGs all along NR PTs. These observations could be related to the down-regulation of eight PMEs and associated to the pulsating-growth of PTs. As such, in Arabidopsis pme48 pollen grains, in which PME activity was reduced and the degree of methylesterification of HGs was higher than in WT, germination was delayed and PT length was reduced (Leroux et al., 2015). On the other hand, in Solanum chacoense, exogenous application of PME to germinating pollen grains induced a reduction of PT growth, an increase of cell wall stiffness and a reduction of its visco-elasticity (Parre and Geitmann, 2005), suggesting that tight regulations of PME activity are required for proper cell wall remodeling and PT growth. The fact that no change in global PME activity was observed among the various lines is not surprising, as temporal and spatial changes revealed by the immunolocalization of methylesterified HG are more refined than the measurement of global activity after crushing whole PTs.

The rate of PT elongation is determined by the equilibrium between turgor-pressure and cell wall ability to extend under this pressure (Kroeger et al, 2011). We observed that PTs exhibited larger diameters in NR than in WTB in liquid medium only. In contrast, in etr3-ko, the diameter of PTs in liquid medium was smaller than in WT. The down-regulation of PMEs in NR presumably did not allow the proper demethylesterification of HGs in the sub-apical dome of the tip and consequently the normal $\mathrm{Ca}^{2+}$ bridging of deesterified $\mathrm{HGs}$, thus decreasing the stiffness of the cell wall in the shank of PTs. This led to an increase of PT diameter under the internal turgor-pressure. This increase was also observed in liquid medium in pme48 (Leroux et al., 2015). These data demonstrate that solid medium can provide a mechanical support to PTs and that the stiffness of the medium can also differently modulate PT growth (Gossot \& Geitmann, 2007; Reimann et al., 2020).

Taken together, our results suggest that ethylene signaling can modify the cell wall structure of PTs by regulating the transcription of genes encoding enzymes involved in pectin and hemicellulose modifications. These changes, especially those observed in the degree of 
methylesterification of HGs, induce a reduction in the length of PTs and an increase in their diameters when ethylene perception is constitutive as in $N R$, and the reverse phenotype when one of the major ETRs present in PTs is inactive like in etr3-ko.

\section{Calcium signaling is a main target of ethylene signaling in pollen tubes}

Tip-focused $\mathrm{Ca}^{2+}$ gradients generated by interplay between $\mathrm{Ca}^{2+}$ pumps and channels are observed during PT elongation and correlated with pulses in growth rates (Pierson et al., 1996; and for a recent review see Zheng et al., 2019). In our study, more than 30 genes coding for proteins involved in $\mathrm{Ca}^{2+}$ homeostasis and decoding were de-regulated in the two ETR mutants. Such a high DEG number indicates the tight link that exists between ethylene and $\mathrm{Ca}^{2+}$ signaling, and highlights the importance of the latter in modulating tomato PT elongation. More than 25 of these DEGs were repressed in NR.

Interestingly, a few DEGs were inversely de-regulated in NR and etr3-ko, in agreement with the observed opposite growth and tip-focused $\mathrm{Ca}^{2+}$ gradient phenotypes. This is the case for the calreticulin gene (Solyc01g100380), which expression is down-regulated in etr3-ko and upregulated in NR. This protein was already shown to be important for $\mathrm{Ca}^{2+}$ homeostasis during PT growth in P. hybrida (Suwinska et al., 2017). Conversely, CPK17 (Solyc12g099790) is upregulated in etr3-ko and down-regulated in NR. CPK17 was shown to be preferentially expressed in Arabidopsis PTs and to have a redundant function with CPK34 during PT growth and tropism (Myers et al., 2009). The PTs of the double mutant cpk17/cpk34 display a slower growth and impaired tropism. The CPK17 ortholog being up-regulated in etr3-ko and down-regulated in NR could fit with longer PTs observed in etr3-ko and shorter PTs observed in NR. Genes encoding two other $\mathrm{Ca}^{2+}$ effectors, CPK20 (Solyc10g076900) and calcineurin B-like protein3 (CBL3) (Solyc07g065820) are also up-regulated in etr3-ko PTs. These two $\mathrm{Ca}^{2+}$ effectors have been reported to be associated with PT growth by activating the slow anion channel SLAH3 at the PT tip (Gutermuth et al., 2013), whereas CBL3 activates CIPK12 to promote a fast PT growth (Steinhorst et al., 2015). The gene (Solyc10g086060) that showed the strongest amplitude between inhibition (in NR) and stimulation (in etr3-ko) is homologous to the Arabidopsis gene At5g03040. This gene encodes the $\mathrm{Ca}^{2+}$-calmodulin binding protein IQD2, of the large IQD family. These family members participate in microtubule organization during plant development and are modulated by auxin (Bürstenbinder et al., 2017). Here, it appears that removing the ETR3, in the LOF, leads to the up-regulation of IQD2 that could promote PT expansion by favoring the cytoskeleton organization required for a proper elongation of the cell.

This article is protected by copyright. All rights reserved 
Regarding $N R$, there were only a few up-regulated genes: two encoding $\mathrm{Ca}^{2+}$-ATPases, calciumtransporting ATPase 4 (ECA4) (Solyc11g072880), ACA9 (Solyc03g123890) and one encoding CML7 (Solyc06g083000). Considering that PTs were shorter in NR than in etr3-ko, we can speculate that these ATPases and CML7 could contribute to dissipate the $\mathrm{Ca}^{2+}$ gradient by extruding the $\mathrm{Ca}^{2+}$ ions from the cytosol. Indeed, in NR, the Fluo-4 fluorescence is systematically lower than in WTB, indicating a lower $\mathrm{Ca}^{2+}$ concentration in PT tips. However, we cannot exclude a membrane permeability disturbance due to the mutation, which would affect the loading of the probe in PTs.

In $N R$, there were down-regulated genes encoding the CMLs 3, 13, 16, 27, 28 and 46 (Solyc05g050750, Solyc11g072270, Solyc01g010020, Solyc06g068960, Solyc02g063340 and Solyc02g067220, respectively) and two genes encoding cyclic nucleotide-gated ion channels, CNGC8 and 16 (Solyc03g116850 and Solyc03g114110, respectively). CNGCs together with iGLUr channels are the main players in generating the $\mathrm{Ca}^{2+}$ influx in PTs (Michard et al., 2017; Gao et al., 2016; Tunc-Ozdemir et al, 2013). CNGC8 was recently shown to be involved in PT growth by antagonizing CNGC18 (Pan et al., 2019). With respect to shorter PTs observed in NR, a reduced activity of these CNGC channels in conjunction with a supposed increased activity of $\mathrm{Ca}^{2+}$-ATPases could result in a disruption/mitigation of the tip-focused $\mathrm{Ca}^{2+}$ gradient as confirmed by the lower Fluo-4 fluorescence observed in PT tips. Concerning CMLs, little is known about their targets in plants but most of the genes cited above have been already shown to be upregulated during pollen germination and/or PT growth (Wang et al 2008). Moreover, it is not excluded that one of these down-regulated genes encode CMLs that could participate in either a positive or a negative regulation of one of the two identified $\mathrm{Ca}^{2+}$-ATPases as already shown (Astegno et al., 2017). In addition, several other genes belonging to the $\mathrm{Ca}^{2+}$ toolkit are also down-regulated including several genes encoding CIPKs or cation exchangers. These are known to be directly involved in the regulation of ions channels governing ion homeostasis during PT growth. The Arabidopsis gene homologous to the down-regulated annexin 5 gene (Solyc04g008270) was shown to be involved in pollen development and PT growth by promoting $\mathrm{Ca}^{2+}$-dependent membrane trafficking necessary for PT elongation (Zhu et al., 2014). The additional experiment, using $\mathrm{La}^{3+}$ to block calcium fluxes, proved that calcium is downstream of the ethylene signal, as no PT elongation was observed in any of the tomato lines in the presence of lanthanum, whatever the signals delivered by ETRs.

Overall, the calcium signaling-related DEGs in the two ETR mutants similarly point out the crucial role of ethylene in controlling the tip-focused $\mathrm{Ca}^{2+}$ gradient required for PT growth. The fact that 
the application of lanthanum inhibits calcium transport in all lines, wild types and loss- or gain-offunction ETR mutants, is a proof that calcium transport is downstream of the ethylene signaling.

In summary (Figure 6F), our work suggests that ETR signaling blocks PT growth, in absence of a sufficient amount of ethylene. This blockade is notably mediated by cell wall modifying enzymes acting on the methylesterification status of $\mathrm{HGs}$, and by an alteration of $\mathrm{Ca}^{2+}$ loading in PTs. Changes in ethylene signaling is also altering expression of other gene families, particularly related to hormone metabolism, that were not detailed in this study. Contributions of the ethylene precursor, 1-aminocyclopropane carboxylic acid, also has been suggested to play crucial roles in ovular PT attraction (Mou et al., 2020). Ethylene has long been known to be involved in many plant development processes, and its contribution to fruit set has been under scrutiny over the last decades (An et al., 2020). Here, we unravel part of its crucial role in PT growth.

By altering ethylene perception, we show that ethylene signal acts on both cell wall- and calciumrelated genes. Specific effects on HG methylesterification and calcium signaling were demonstrated.

\section{EXPERIMENTAL PROCEDURES}

\section{Plant material and growth conditions}

Tomato lines (Solanum lycopersicum cv MicroTom): the etr3-ko and etr4-ko mutant lines were generated in our laboratory using the CRISPR-Cas9 technology, as described (Chen et al., 2020a), these ko lines are Loss-Of-Function (LOF). Briefly the sgRNAs "GAATCCTGTGATTGCATTGAGG" and "GCGATGTAACTGTGATGATGAGG" were designed to knock out ETR3 and ETR4, respectively. The etr3-ko used in this study has an adenine missing at the $4^{\text {th }}$ position before the PAM (protospacer adjacent motif) resulting in a frameshift mutation. For etr4-ko, a guanine was deleted at the $4^{\text {th }}$ position before the PAM. The comparisons of the amino acid sequences of the mutated ETR proteins in etr3-ko and etr4-ko to their wild types are shown in Figure S8. Unfortunately, we did not succeed in the mutagenesis of ETR5. WTB and NR mutant lines were obtained from the L.E. Pereira Peres laboratory (Carvalho et al., 2011), the $N R$ line is a Gain-Of-Function line (GOF), due to a point-mutation in ETR3. All lines are MicroTom cultivar, but WTB and NR might have evolved slightly differently over the last 20 years since they were distributed by the Avi Levy's laboratory (Weizmann Institute, Israel). Thus, we used two wild-type lines, WT and WTB. WT is the wild type we used in the laboratory over the last 20 years, with which we performed the CRISPR-Cas9 constructs for etr-ko. In WTB, the "B" stands for Brazil. Plant sowing and growing conditions are detailed in Chen et al. (2020a). 


\section{In vitro pollen grain germination assay}

For germination assays, pollen was sampled from flowers at one and two days post-anthesis (DPA) (Figure S9), at second and third positions of flower clusters. Pollen grains from 10 anthers were collected in $1.5 \mathrm{~mL}$ centrifuge tubes using an electric toothbrush body (Oral-B, France). The germination medium (GM) as in Firon et al. (2012), containing $0.5 \%$ (w/v) agarose or not. Pollen grains were spread over solid GM or suspended in $0.5 \mathrm{~mL}$ of liquid $\mathrm{GM}$, and incubated at $25^{\circ} \mathrm{C}$ for various times in the dark. Images were acquired with an inverted microscope (Leitz DMIRB, Leica Microsystems, Germany) equipped with a 10M pixel CMOS camera (MC 190HD, Leica), with a 10x/0.22NA NPLAN objective (Leica). PT length and diameter were measured with ImageJ (Abràmoff et al., 2004). The lanthanum was applied at $50 \mu \mathrm{M} \mathrm{LaCl}$ in liquid $\mathrm{GM}$ as described in Qu et al. (2016).

\section{Ethylene and 1-MCP}

Pollen grains were spread on solid GM as described above, the Petri dishes were sealed with an electric tape, and ethylene or 1-MCP were injected in the headspace at the desired concentration, and incubated for $4 \mathrm{~h}$ before germination or PT length measurements. Preliminary experiments were performed to validate optimal concentrations, as in Hen et al. (2020). Endogenous ethylene measurements by PTs were performed by sampling the headspace in a $2 \mathrm{~mL}$ brown glass vials containing around $10 \mathrm{mg}$ of pollen grains in $0.5 \mathrm{~mL}$ of liquid $\mathrm{GM}, 4 \mathrm{~h}$ after imbibition, and analysing the headspace gas sample as previously detailed (Chen et al., 2020b). A vial with GM only was used as control. Numbers of pollen grains per $\mu \mathrm{L}$ were assessed by microscopy.

\section{RNA extraction and RT-qPCR}

Pollen grains $\left(20 \mathrm{mg}\right.$ ) were suspended $0.5 \mathrm{~mL}$ of liquid $\mathrm{GM}$. After $4 \mathrm{~h}$ of germination at $25^{\circ} \mathrm{C}$ in the dark, PTs were centrifuged at $16,000 \mathrm{~g}$ for $5 \mathrm{~min}$. The supernatant was removed and the pellet was frozen into liquid nitrogen and stored at $-80^{\circ} \mathrm{C}$. Samples were ground with a ball grinder using Tissue Lyser II (Qiagen, France) for $1 \mathrm{~min}$. The pollen powder (20 $\mathrm{mg}$ ) was used to extract total RNA using the ReliaPrep ${ }^{\mathrm{TM}}$ RNA Tissue Miniprep System kit (Promega, France) and treated with DNase I (Invitrogen, France). RT-qPCR analyses were performed as described (Chen et al., 2020a). Primers are listed in Table S1. ETR6 transcripts were always below detection threshold. Thus, ETR6 was not used in correlation analysis between RNA-seq reads and RT-qPCR results. 


\section{RNA-seq analyses}

For each line (WT, WTB, etr3-ko and NR), four biological replicates were prepared, and RNA quality was checked on Agilent 2100 Bioanalyzer System, using the RNA 6000 Nano kit protocol (Agilent Technologies, Germany). RNA samples with RNA Integrity Number (RIN) values greater than 7.5 were sent to the GENEWIZ company (Germany) for sequencing (standard service, 2x150bp, HiSeq Illumina Platform). Raw sequenced reads were treated using the pipeline described in Figure S10, implemented inside a data-driven computational pipeline called NextFlow (Di Tommaso et al., 2017): raw sequenced reads in Fastq format were cleaned after quality check using fastQC version 0.11 .05 (Andrews, 2010). To use only high-quality sequences, reads were cleaned by removing all left sequencing adaptors and by trimming low quality bases.

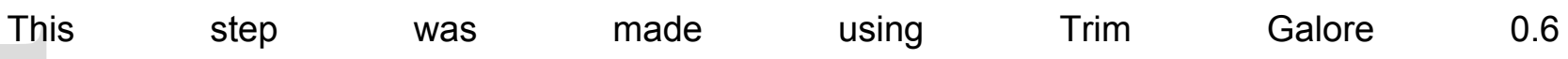
(http://www.bioinformatics.babraham.ac.uk/projects/trim_galore/), a wrapping tool based on Cutadapt (Martin, 2011). Cleaned sequences were mapped to an improved reference tomato genome produced and assembled by the GBF lab with the annotation SImic1.1 (http://tomatogenome.gbfwebtools.fr/) using STAR 2.5.1b, a spliced-aware mapper (Dobin et al., 2013). The mapping parameters took into account each library size when paired-end sequencing was performed. Finally, featureCounts 1.6.0 (Liao et al., 2014) was used to calculate read counts for each gene from the mapping file. The homologies between SImic 1.1 (Sly nomenclature) and ITAG 2.4 (Solyc) annotation (https://solgenomics.net/organism/Solanum_lycopersicum/) is available at http://tomatogenome.gbfwebtools.fr/correspondence, and in Table S3A. The homologies between SImic 1.1 and ITAG 4.1 are available in Table S3B, and all RNA sequences are available from the European Nucleotide Archive, accession number E-MTAB-9660.

\section{Differential expression and downstream analyses}

Without a priori knowledge of gene expression variances, we optimized our experimental design with four biological replicates for each condition of interest (Lamarre et al., 2018). Differential expression (DE) analysis was performed with $R$ software using DESeq2 package with default Relative Log Expression (RLE) normalization method, encompassing biases caused by library size and relative size of transcriptomes (Love et al., 2014; Maza et al., 2013, Maza, 2016). False discovery rate was limited by using a threshold for adjusted p-values equal to 0.05 (Benjamini and Hochberg, 1995). To visualize distances between samples, a PCA of normalized expressions was performed with plotPCA function (DESeq2 package), to check the homogeneity of the biological replicates and relative distances between conditions. The RLE normalization method used in DESeq2 for the DE analysis, considering that transcripts have the same length in all 
conditions, do not normalize counts by transcript length. Thus, normalized counts generated by DESeq2 need to be normalized by transcript length to allow comparison between transcript levels within a condition. Then, DESeq2 normalized samples were multiplied by 100 (rough magnitude of read lengths, in bp) and divided by the transcript length (in bp). Finally, the mean of the biological replicates for a given condition was calculated. This mean relates to the number of transcripts produced in this condition (Zouine et al., 2017). Venn diagrams were generated with JVENN software (http://jvenn.toulouse.inra.fr/app/example.html). The RNA-seq results are available in Table S3.

The MAPMAN annotation (https://mapman.gabipd.org/mapman) and the ProtAnnDB (http://www.polebio.Irsv.ups-tlse.fr/ProtAnnDB/) tools were used to identify functional categories of genes and to determine genes that could be related to calcium signaling and cell wall modification. TAIR 11 annotation was selected to search for homologous genes in Arabidopsis by using BLAST+ tool, version 2.9.0. The calcium signaling-related genes are listed in Table S5. The proteins predicted to be secreted and having no more than one transmembrane domain and no intracellular retention signal have been selected as cell-wall related proteins (Table S4). They have been named following the nomenclature of WallProtDB, a database dedicated to cell wall proteins identified in cell wall proteomes (San Clemente and Jamet, 2015) and the CAZy database (http://www.cazy.org/).

\section{Pectin immunolabeling and PME activity measurement}

The immunolabeling was performed as described (Hocq et al., 2020). Briefly, after $4 \mathrm{~h}$ of growth in liquid GM, PTs were treated with a fixation medium: $100 \mathrm{mM}$ PIPES, $4 \mathrm{mM} \mathrm{MgSO} \mathrm{m}_{4}, 7 \mathrm{H}_{2} \mathrm{O}, 4$ mM EGTA, $10 \%(\mathrm{w} / \mathrm{v})$ sucrose, and $5 \%$ of paraformaldehyde $\mathrm{pH} 7.5$ and stored at $4{ }^{\circ} \mathrm{C}$ until use. The fixed PTs were centrifuged at $4,000 \mathrm{~g}$ for $7 \mathrm{~min}$ and washed 3 times with $250 \mu \mathrm{L}$ PBS, pH 7.4 , incubated in a blocking solution (PBS containing $3 \%$ fat-free milk) for $30 \mathrm{~min}$ at room temperature. After three washes, PTs were incubated overnight with the primary antibody, LM19 or LM20, for weakly or highly methylesterified HGs, respectively (PlantProbes, http://www.plantprobes.net/) (Verhertbruggen et al., 2009) at $1: 5$ dilution in PBS at $4^{\circ} \mathrm{C}$ in the dark. After washing the pellet 3 times, it was incubated for $3 \mathrm{~h}$ at $30^{\circ} \mathrm{C}$ in the dark with the secondary Alexa488 anti-rat antibody at 1:50 dilution in PBS, followed by 3 washes with PBS. Controls were incubated with the secondary antibody only. Images were acquired with a Leica DMI6000 B inverted microscope equipped with DFC 450C camera and LAS V3.8 software. Alexa488 was detected with a filter cube $L 5, \lambda$ excitation at $460-500 \mathrm{~nm}$ and $\lambda$ emission at 510 $540 \mathrm{~nm}$. All the samples were observed at the same exposure time. 
For PME assays, the protein extraction from PT was carried out with $2 \mathrm{M} \mathrm{NaCl}$ buffer according to Baldwin et al. (2014). After desalting, protein concentration was measured with the Bradford assay (Bio-Rad, France). The total PME activity was assayed according to Baldwin et al. (2014) by quantifying the amount of methanol released from citrus pectin with a degree of methylesterification $>85 \%$ (Sigma-Aldrich, France). The mean \pm SD of PME activity is from 3 biological replicates.

\section{Intracellular calcium detection and imaging}

Intracellular $\mathrm{Ca}^{2+}$ was detected using Fluo-4 AcetoxyMethyl ester (Fluo-4/AM, Invitrogen, USA) as described (Qu et al., 2015) with the following adjustments. Stock aliquots of fluo-4/AM were made of $50 \mu \mathrm{g}$ fluo-4/AM dissolved in $91.2 \mu \mathrm{L}$ anhydrous dimethyl sulfoxide solution, stored at $-20^{\circ} \mathrm{C}$. PTs were imbibed for $1 \mathrm{~h}$ in liquid GM at a concentration of $8 \times 10^{5}$ cells $\cdot \mathrm{mL}^{-1}$, treated with a final concentration of $1 \mu \mathrm{M}$ fluo-4/AM, and incubated for $30 \mathrm{~min}$ at $22^{\circ} \mathrm{C}$ in the dark. Images were acquired with CLSM confocal microscope Leica SP8, driven by LAS $X$ software, with water immersion objective 25x/0.95. Fluo-4/AM was excited at $488 \mathrm{~nm}$ and fluorescence was collected on photomultiplier between 500 and $690 \mathrm{~nm}$. Images were acquired on one plan, every $2 \mathrm{~s}$ for a $30 \mathrm{~min}$ total duration. The maximum fluorescence intensity observed over the time course, for one PT apex, was selected for quantification with ImageJ (Abràmoff et al., 2004), using the mean grey value, over a constant area, shown in figures with a yellow dashed line. The mean of maximum fluorescence of 10 individual PTs for each genotype was measured.

\section{DATA AVAILABILITY}

All RNA-seq data were deposited in the European Nucleotide Archive under the accession number E-MTAB-9660.

\section{ACKNOWLEDGMENTS}

We thank Mondher Bouzayen and Mohamed Zouine, head and co-head of GBF lab. We are grateful to Yves Martinez and Aurélie Le Ru at the FR AIB imaging platform, for help with microscopy. We are grateful to Marc Ropitaux for the training at Glyco-MEV in Rouen and to Dr Catherine Rayon for relevant advises regarding the PME activity determination. Thanks to the RAA's PhD committee, including Caren Chang (Univ. Maryland), Nicolas Frei-dit-Frey (Univ. Toulouse), and Huguette Sallanon (Univ. Avignon) for fruitful discussions. EJ is thankful to CNRS 
and the Toulouse 3 University for supporting her work. The authors declare no competing interests.

\section{FUNDING}

This work was funded by a Toulouse INP PhD grant to RAA, by FR AIB and TTIL Toulouse INP grants to CC, and partially by a CSC PhD grant to YC, and by TomGEM H2020 EU project (679796) and by the TomEpiSet ANR project.

\section{AUTHOR CONTRIBUTIONS}

RAA and CC conceived the study, YC and RAA performed the CRISPR mutations, and RAA, PF, EM and AD performed the RNA-seq. RAA, CM, EJ and JCM managed experiments and analyses related to calcium, pollen culture and cell wall. All co-authors wrote and approved the manuscript.

\section{CONFLICT OF INTEREST}

The authors declare no conflict of interest.

\section{SUPPORTING INFORMATION}

Figure S1. Flower morphology of Solanum lycopersicum (cv. MicroTom).

Figure S2. Tomato pollen tube (PT) growth as a function of time after imbibition.

Figure S3. Quality indicators of RNA-seq experiment.

Figure S4. Expression levels of PME genes, and calcium related genes in germinating tomato pollen grains.

Figure S5. Immunolabeling of weakly methylesterified HG epitopes probed with LM19, and PME acitivity.

Figure S6. $\mathrm{Ca}^{2+}$ gradients in apical regions of pollen tubes and lanthanum, ethylene and 1-MCP trials.

Figure S7. Effects of ethylene and 1-MCP concentrations on the rate of pollen germination and the pollen tube growth of WT

Figure S8. etr3-ko and etr4-ko tomato protein sequences.

Figure S9. Pollen viability at different stages of flower development.

Figure S10. Overview of the RNA-Seq analysis pipeline.

Table S1. Genes and primer sequences used for RT-qPCR analyses.

Table S2. Correlation between RNAseq and RT-qPCR data, and feedback of ETR mutations on ETR expressions 
Table S3. Global list of sequenced RNAs (tab A), correspondence between the Sly and Solyc of ITAG 4.1 (tab B).

Table S4. Comparison between the levels of expression of cell wall-related genes in the four tomato lines.

Table S5. Comparison between the levels of expression of calcium signaling-related genes in the four tomato lines.

\section{REFERENCES}

Abràmoff, M.D., Magalhães, P.J., and Ram, S.J. (2004). Image Processing with ImageJ. Biophotonics Int., 11, 36-42

An, J., Althiab Almasaud, R., Bouzayen, M., Zouine, M., and Chervin, C. (2020). Auxin and ethylene regulation of fruit set. Plant Sci. 292, 110381

Andrews, S. 2010. FastQC: A quality control tool for high throughput sequence data [Online]. http://www.bioinformatics.babraham.ac.uk/projects/fastqc/

Astegno, A., Bonza, M.C., Vallone, R., La Verde, V., D’Onofrio, M., Luoni, L., Molesini, B., and Dominici, P. (2017). Arabidopsis calmodulin-like protein CML36 is a calcium $\left(\mathrm{Ca}^{2+}\right)$ sensor that interacts with the plasma membrane $\mathrm{Ca}^{2+}$-ATPase isoform ACA8 and stimulates its activity. J. Biol. Chem. 292, 15049-15061.

Baldwin, L., Domon J.M., Klimek J.F., Fournet F., Sellier H., Gillet F., Pelloux J., Lejeune-Hénaut I., Carpita N.C., Rayon C. (2014). Structural alteration of cell wall pectins accompanies pea development in response to cold. Phytochemistry. 104, 37-47.

Benjamini, Y., and Hochberg, Y. (1995). Controlling the false discovery rate: a practical and powerful approach to multiple testing. J. R. Stat. Soc. Ser. B 57, 289-300.

Binder, B. M. (2020). Ethylene signaling in plants. J. Biol. Chem. 295, 7710-7725.

Bisson, M.M.A., and Groth, G. (2015). Targeting plant ethylene responses by controlling essential protein-protein interactions in the ethylene pathway. Mol. Plant 8, 1165-1174.

Bosch, M., Cheung, A.Y., Hepler, P.K. (2005). Pectin methylesterase, a regulator of pollen tube growth. Plant Physiol. 138, 1334-1346.

Brummell, D.A. (2006). Cell wall disassembly in ripening fruit. Funct. Plant Biol. 33, 103. 
Bürstenbinder, K., Mitra, D., and Quegwer, J. (2017). Functions of IQD proteins as hubs in cellular calcium and auxin signaling: A toolbox for shape formation and tissue-specification in plants? Plant Signal. Behav. 12, e1331198.

Carvalho, R.F., Campos, M.L., Pino, L.E., Crestana, S.L., Zsögön, A., Lima, J.E., Benedito, V.A., and Peres, L.E.P. (2011). Convergence of developmental mutants into a single tomato model system: "Micro-Tom" as an effective toolkit for plant development research. Plant Methods 7 , $1-14$.

Chang, C. (2016). Q and A: How do plants respond to ethylene and what is its importance? BMC Biol. 14, 1-7.

Chebli, Y., Kaneda, M., Zerzour, R., and Geitmann, A. (2012). The cell wall of the Arabidopsis pollen tube-spatial distribution, recycling, and network formation of polysaccharides. Plant Physiol. 160, 1940-1955.

Chen, Y., Rofidal, V., Hem, S., Gil, J., Nosarzewska, J., Berger, N., Demolombe, V., Bouzayen, M., Azhar, B.J., Shakeel, S.N., et al. (2019). Targeted proteomics allows quantification of ethylene receptors and reveals SIETR3 accumulation in Never-Ripe tomatoes. Front. Plant Sci. 10, 1-10.

Chen, Y., Hu, G., Rodriguez, C., Liu, M., Binder, B.M., and Chervin, C. (2020a). Roles of SIETR7, a newly discovered ethylene receptor, in tomato plant and fruit development. Hortic. Res. 7, 17.

Chen, Y., Althiab Almasaud, R., Carrie, E., Desbrosses, G., Binder, B.M., and Chervin, C. (2020b). Ethanol, at physiological concentrations, affects ethylene sensing in tomato germinating seeds and seedlings. Plant Sci. 291, 110368.

Dardelle, F., Lehner, A., Ramdani, Y., Bardor, M., Lerouge, P., Driouich, A., and Mollet, J.-C. (2010). Biochemical and immunocytological characterizations of Arabidopsis pollen tube cell wall. Plant Physiol. 153, 1563-1576.

Dearnaley, J.D.W., and Daggard, G.A. (2001). Expression of a polygalacturonase enzyme in germinating pollen of Brassica napus. Sex. Plant Reprod. 13, 265-271.

Dehors, J., Mareck, A., Kiefer-Meyer, M.-C., Menu-Bouaouiche, L., Lehner, A., and Mollet, J.-C. (2019). Evolution of cell wall polymers in tip-growing land plant gametophytes: composition, distribution, functional aspects and their remodeling. Front. Plant Sci. 10, 1-28.

Di Tommaso, P., Chatzou, M., Floden, E. W., Barja, P. P., Palumbo, E., and Notredame, C. (2017). Nextflow enables reproducible computational workflows. Nat. Biotechnol. 35, 316-319. 
Dobin, A., Davis, C. A., Schlesinger, F., Drenkow, J., Zaleski, C., Jha, S., Batut, P., Chaisson, M., and Gingeras, T.R. (2013). STAR: Ultrafast universal RNA-seq aligner. Bioinformatics 29, 15-21.

Firon, N., Pressman, E., Meir, S., Khoury, R., and Altahan, L. (2012). Ethylene is involved in maintaining tomato (Solanum lycopersicum) pollen quality under heat-stress conditions. $A o B$ Plants 2012, pls024.

Gao, Q.F., Gu, L.L., Wang, H.Q., Fei, C.F., Fang, X., Hussain, J., Sun, S.J., Dong, J.Y., Liu, H., and Wang, Y.F. (2016). Cyclic nucleotide-gated channel 18 is an essential $\mathrm{Ca}^{2+}$ channel in pollen tube tips for pollen tube guidance to ovules in Arabidopsis. Proc. Natl. Acad. Sci. U. S. A. 113, 3096-3101.

Geitmann, A. (2010). How to shape a cylinder: Pollen tube as a model system for the generation of complex cellular geometry. Sex. Plant Reprod. 23, 63-71.

Giovane, A., Servillo, L., Balestrieri, C., Raiola, A., D’Avino, R., Tamburrini, M., Ciardiello, M. A., and Camardella, L. (2004). Pectin methylesterase inhibitor. Biochim. Biophys. Acta 1696, 245-252.

Gossot, O., and Geitmann, A. (2007). Pollen tube growth: coping with mechanical obstacles involves the cytoskeleton. Planta 226, 405-416.

Gustavsson, J., Cederberg, C. and Sonesson, U. (2011). Global food losses and food waste. FAO report, Rome, http://www.fao.org/3/a-i2697e.pdf.

Gutermuth, T., Lassig, R., Portes, M., Maierhofer, T., Romeis, T., Borst, J., Hedrich, R., and Konrad, K. R. (2013). Pollen tube growth regulation by free anions depends on the interaction between the anion channel SLAH3 and calcium-dependent protein kinases CPK2 and CPK20. Plant Cell 25, 4525-4543.

Hepler, P.K., Kunkel, J.G., Rounds, C.M., and Winship, L.J. (2012). Calcium entry into pollen tubes. Trends Plant Sci. 17, 32-38.

Hepler, P.K., Rounds, C.M., and Winship, L.J. (2013). Control of cell wall extensibility during pollen tube growth. Mol. Plant 6, 998-1017.

Hijazi, M., Velasquez, S.M., Jamet, E., Estevez, J.M., and Albenne, C. (2014). An update on posttranslational modifications of hydroxyproline-rich glycoproteins: Toward a model highlighting their contribution to plant cell wall architecture. Front. Plant Sci. 5, 1-10. 
Hocq, L., Pelloux, J., and Lefebvre, V. (2017). Connecting homogalacturonan-type pectin remodeling to acid growth. Trends Plant Sci. 22, 20-29.

Hocq, L., Guinand, S., Habrylo, O., Voxeur, A., Tabi, W., Safran, J., Fournet, F., Domon, J.M., Mollet, J.-C., Pilard, S., et al. (2020). The exogenous application of AtPGLR, an endopolygalacturonase, triggers pollen tube burst and repair. Plant J. 103, 617-633.

Holden, M.J., Marty, J. A., and Singh-Cundy, A. (2003). Pollination-induced ethylene promotes the early phase of pollen tube growth in Petunia inflata. J. Plant Physiol. 160, 261-269.

Jegadeesan, S., Beery, A., Altahan, L., Meir, S., Pressman, E., and Firon, N. (2018). Ethylene production and signaling in tomato (Solanum lycopersicum) pollen grains is responsive to heat stress conditions. Plant Reprod. 31, 367-383.

Jia, H., Yang, J., Liesche, J., Liu, X., Hu, Y., Si, W., Guo, J., and Li, J. (2018). Ethylene promotes pollen tube growth by affecting actin filament organization via the cGMP-dependent pathway in Arabidopsis thaliana. Protoplasma 255, 273-284.

Ju, C., Yoon, G. M., Shemansky, J. M., Lin, D.Y., Ying, Z.I., Chang, J., Garrett, W.M., Kessenbrock, M., Groth, G., Tucker, M.L., et al. (2012). CTR1 phosphorylates the central regulator EIN2 to control ethylene hormone signaling from the ER membrane to the nucleus in Arabidopsis. Proc. Natl. Acad. Sci. U. S. A. 109, 19486-19491.

Jung, T., Lee, J.H., Cho, M.H., and Kim, W.T. (2000). Induction of 1-aminocyclopropane-1carboxylate oxidase mRNA by ethylene in mung bean roots: Possible involvement of $\mathrm{Ca}^{2+}$ and phosphoinositides in ethylene signalling. Plant Cell Environ. 23, 205-213.

Kroeger, J. H., Zerzour, R., and Geitmann, A. (2011). Regulator or driving force? The role of turgor pressure in oscillatory plant cell growth. PLoS One 6, e18549.

Lamarre, S., Frasse, P., Zouine, M., Labourdette, D., Sainderichin, E., Hu, G., Le Berre-Anton, V., Bouzayen, M., and Maza, E. (2018). Optimization of an RNA-Seq differential gene expression analysis depending on biological replicate number and library size. Front. Plant Sci. 9, 108

Leroux, C., Bouton, S., Kiefer-Meyer, M.C., Fabrice, T.N., Mareck, A., Guénin, S., Fournet, F., Ringli, C., Pelloux, J., Driouich, A., et al. (2015). PECTIN METHYLESTERASE48 is involved in arabidopsis pollen grain germination. Plant Physiol. 167, 367-380.

Li, S., Han, X., Yang, L., Deng, X., Wu, H., Zhang, M., Liu, Y., Zhang, S., and Xu, J. (2018). Mitogen-activated protein kinases and calcium-dependent protein kinases are involved in 
wounding-induced ethylene biosynthesis in Arabidopsis thaliana. Plant Cell Environ. 41, 134147.

Liao, Y., Smyth, G.K., and Shi, W. (2014). FeatureCounts: An efficient general purpose program for assigning sequence reads to genomic features. Bioinformatics 30, 923-930.

Llop-Tous, I., Barry, C.S., and Grierson, D. (2000). Regulation of ethylene biosynthesis in response to pollination in tomato flowers. Plant Physiol. 123, 971-978.

Love, M. I., Huber, W., and Anders, S. (2014). Moderated estimation of fold change and dispersion for RNA-seq data with DESeq2. Genome Biol. 15, 1-21.

Malhó, R., and Trewavas, A.J. (1996). Localized apical increases of cytosolic free calcium control pollen tube orientation. Plant Cell 8, 1935-1949.

Martin, M. 2011. Cutadapt removes adapter sequences from high-throughput sequencing reads. EMBnet 17, 10-12

Maza, E. (2016). In papyro comparison of TMM (edgeR), RLE (DESeq2), and MRN normalization methods for a simple two-conditions-without-replicates RNA-seq experimental design. Front. Genet. 7, 1-8.

Maza, E., Frasse, P., Senin, P., Bouzayen, M., and Zouine, M. (2013). Comparison of normalization methods for differential gene expression analysis in RNA-Seq experiments. Commun. Integr. Biol. 6, e25849.

Michard, E., Simon, A.A., Tavares, B., Wudick, M.M., and Feijó, J.A. (2017). Signaling with ions: The keystone for apical cell growth and morphogenesis in pollen tubes. Plant Physiol. 173, 91-111.

Mollet, J.-C., Leroux, C., Dardelle, F., and Lehner, A. (2013). Cell wall composition, biosynthesis and remodeling during pollen tube growth. Plants 2, 107-147.

Mou, W., Kao, Y.-T., Michard, E., Simon, A. A., Li, D., Wudick, M. M., Lizzio, M. A., Feijó, J. A., and Chang, C. (2020). Ethylene-independent signaling by the ethylene precursor ACC in Arabidopsis ovular pollen tube attraction. Nat. Commun. 11, 4082.

Myers, C., Romanowsky, S. M., Barron, Y. D., Garg, S., Azuse, C. L., Curran, A., Davis, R. M., Hatton, J., Harmon, A. C., and Harper, J. F. (2009). Calcium-dependent protein kinases regulate polarized tip growth in pollen tubes. Plant J. 59, 528-539. 
Pan, Y., Chai, X., Gao, Q., Zhou, L., Zhang, S., Li, L., and Luan, S. (2019). Dynamic interactions of plant CNGC subunits and calmodulins drive oscillatory $\mathrm{Ca}^{2+}$ channel activities. Dev. Cell 48, 710-725.

Parre, E., and Geitmann, A. (2005). Pectin and the role of the physical properties of the cell wall in pollen tube growth of Solanum chacoense. Planta 220, 582-592.

Petruzzelli, L., Sturaro, M., Mainieri, D., and Leubner-Metzger, G. (2003). Calcium requirement for ethylene-dependent responses involving 1-aminocyclopropane-1-carboxylic acid oxidase in radicle tissues of germinated pea seeds. Plant Cell Environ. 26, 661-671.

Pierson, E. S., Miller, D. D., Callaham, D. A., Van Aken, J., Hackett, G., and Hepler, P. K. (1996). Tip-localized calcium entry fluctuates during pollen tube growth. Dev. Biol. 174, 160-173.

Qu, H.Y., Shang, Z.L., Zhang, S.L., Liu, L.M., and Wu, J.Y. (2007). Identification of hyperpolarization-activated calcium channels in apical pollen tubes of Pyrus pyrifolia. New Phytol. 174, 524-536.

Qu, H., Xing, W., Wu, F., and Wang, Y. (2016). Rapid and inexpensive method of loading fluorescent dye into pollen tubes and root hairs. PLoS One 11, e0152320.

Reimann, R., Kah, D., Mark, C., Dettmer, J., Reimann, T. M., Gerum, R. C., Geitmann, A., Fabry, B., Dietrich, P., and Kost, B. (2020). Durotropic growth of pollen tubes. Plant Physiol. 183, 558-569.

San Clemente, H., and Jamet, E. (2015). WallProtDB, a database resource for plant cell wall proteomics. Plant Meth. 11, 2.

Schwacke, R., Ponce-Soto, G. Y., Krause, K., Bolger, A. M., Arsova, B., Hallab, A., Gruden, K., Stitt, M., Bolger, M.E., and Usadel, B. (2019). MapMan4: A refined protein classification and annotation framework applicable to multi-omics data analysis. Mol. Plant 12, 879-892.

Steinhorst, L., Mähs, A., Ischebeck, T., Zhang, C., Zhang, X., Arendt, S., Schültke, S., Heilmann, I., and Kudla, J. (2015). Vacuolar CBL-CIPK12 $\mathrm{Ca}^{2+}$-sensor-kinase complexes are required for polarized pollen tube growth. Curr. Biol. 25, 1475-1482.

Stranne, M., Ren, Y., Fimognari, L., Birdseye, D., Yan, J., Bardor, M., Mollet, J.-C., Komatsu, T., Kikuchi, J., Scheller, H.V., et al. (2018). TBL10 is required for O-acetylation of pectic rhamnogalacturonan-I in Arabidopsis thaliana. Plant J. 96, 772-785. 
Suwińska, A., Wasąg, P., Zakrzewski, P., Lenartowska, M., and Lenartowski, R. (2017). Calreticulin is required for calcium homeostasis and proper pollen tube tip growth in Petunia. Planta 245, 909-926.

Tunc-Ozdemir, M., Rato, C., Brown, E., Rogers, S., Mooneyham, A., Frietsch, S., Myers, C.T., Poulsen, L. R., Malhó, R., and Harper, J.F. (2013). Cyclic nucleotide gated channels 7 and 8 are essential for male reproductive fertility. PLoS One 8, e55277.

Verhertbruggen, Y., Marcus, S.E., Haeger, A., Ordaz-Ortiz, J.J., and Knox, J.P. (2009). An extended set of monoclonal antibodies to pectic homogalacturonan. Carbohydr. Res. 344, 1858-1862.

Vogel, J.P., Raab, T. K., Somerville, C.R., and Somerville, S.C. (2004). Mutations in PMR5 result in powdery mildew resistance and altered cell wall composition. Plant J. 40, 968-978.

Vogler, H., Draeger, C., Weber, A., Felekis, D., Eichenberger, C., Routier-Kierzkowska, A.-L., Boisson-Dernier, A., Ringli, C., Nelson, B.J., Smith, R.S., et al. (2013). The pollen tube: a soft shell with a hard core. Plant J. 73, 617-627.

Wang, Y., Zhang, W.Z., Song, L.F., Zou, J. J., Su, Z., and Wu, W.H. (2008). Transcriptome analyses show changes in gene expression to accompany pollen germination and tube growth in Arabidopsis. Plant Physiol. 148, 1201-1211.

Wang, X., Zeng, W., Ding, Y., Wang, Y., Niu, L., Yao, J.L., Pan, L., Lu, Z., Cui, G., Li, G., et al. (2019). Peach ethylene response factor PpeERF2 represses the expression of $A B A$ biosynthesis and cell wall degradation genes during fruit ripening. Plant Sci. 283, 116-126.

Yin, X.R., Allan, A.C., Chen, K.S., and Ferguson, I.B. (2010). Kiwifruit EIL and ERF genes involved in regulating fruit ripening. Plant Physiol. 153, 1280-1292.

Yu, J., Niu, L., Yu, J., Liao, W., Xie, J., Lv, J., Feng, Z., Hu, L., and Dawuda, M. (2019). The involvement of ethylene in calcium-induced adventitious root formation in cucumber under salt stress. Int. J. Mol. Sci. 20, 1047.

Zhao, M.G., Tian, Q.Y., and Zhang, W.H. (2007). Ethylene activates a plasma membrane $\mathrm{Ca}^{2+}$ permeable channel in tobacco suspension cells: Rapid report. New Phytol. 174, 507-515.

Zheng, R.H., Su, S. De, Xiao, H., and Tian, H.Q. (2019). Calcium: a critical factor in pollen germination and tube elongation. Int. J. Mol. Sci. 20, 420. 
Zhu, J., Wu, X., Yuan, S., Qian, D., Nan, Q., An, L., and Xiang, Y. (2014). Annexin5 plays a vital role in Arabidopsis pollen development via $\mathrm{Ca}^{2+}$-dependent membrane trafficking. PLoS One 9, e102407.

Zouine, M., Maza, E., Djari, A., Lauvernier, M., Frasse, P., Smouni, A., Pirrello, J., and Bouzayen, M. (2017). TomExpress, a unified tomato RNA-Seq platform for visualization of expression data, clustering and correlation networks. Plant J. 92, 727-735.

\section{FIGURE CAPTIONS}

\section{Figure 1. Regulation of germinating pollen grains by ethylene}

(A) Expression levels of ETR genes in germinating tomato pollen grains. Total RNA was extracted from WT PTs $4 \mathrm{~h}$ after pollen harvest and imbibition, $\mathrm{n}=4$ biological replicates, LOD stands for lower limit of detection, Relative expression was calculated with regard to ETR1. The sequences of the gene-specific primers used for PCR are listed in Table S1. (B) PT length in WT, WTB, NR and etr3-ko, $4 \mathrm{~h}$ after pollen grain imbibition; and effects of $100 \mathrm{ppm}$ ethylene and 2 ppm 1-MCP treatments on PT length. $n=90$ PTs. (C) Ethylene production by germinating pollen grains, $4 \mathrm{~h}$ after pollen grain imbibition, $\mathrm{n}=6$ biological replicates. For all panels, error bars show SE, different small letters indicate a significant difference between means at $P=0.05$ (Tukey's HSD for panels A and B, Dunn's test for panel C).

Figure 2. Transcriptome profiling of etr3-ko and NR PTs.

(A) PCA of normalized samples. Axes 1 and 2 represent $68 \%$ of data information. Colored dots underline the four conditions of interest and the four biological replicates analysed by RNAseq. See Figure S3 for additional RNAseq quality indicators. (B) Venn diagrams showing the numbers of differentially expressed genes (DEGs) in NR vs WTB and etr3-ko vs WT, up-regulated DEGs, and down-regulated DEGs, base means of normalized counts $>5$, and adjusted P-value $<0.05$. (C) ETRs are differentially expressed in etr3-ko and NR. Bar plot representing log2(fold change) shows differences in expression level between (i) NR vs WTB, and (ii) etr3-ko vs WT, * shows significant changes at $P<0.05$ using Tukey HSD. (D) Functional classification of DEGs. The down-regulated genes are in blue (log2 fold change $<0, P<0.05$ ), and the up-regulated genes in red (log2 fold change $>0, P<0.05)$. Four biological replicates were analyzed.

Figure 3. Changes in the level of transcript accumulation of genes possibly related to pectin modifications, in NR (upper part) and etr3-ko (lower part) compared to WTB and WT, 
respectively. These bar plots represent log2 (fold change) for the pectin-related DEGs $(P<0.05)$. They are grouped in different families: PG (polygalacturonases), PME (pectin methylesterases), PAE (pectin acetylesterases), PL (pectate lyases), PMEI (PME inhibitors), TBL (trichome birefringence-like) and PAC (Proline-rich Arabinogalactan proteins conserved Cysteines). The pectin-related genes are listed in Table S4C.

Figure 4. Changes in the level of transcripts related to calcium signaling, in $N R$ (upper part) and etr3-ko (lower part) compared to WTB and WT, respectively These bar plots represent log2 (fold change) for the calcium-related DEGs $(P<0.05)$. The genes are grouped in families: CIPKs (CBL-interacting protein kinases), CPKs (calcium-dependent protein kinases), CaMBPs (calmodulin binding proteins), CaBPs (calcium-binding proteins), CMLs (calmodulin-like proteins). The calcium signaling-related genes are listed in Table S5.

Figure 5. Immunolocalization of highly methylesterified homogalacturonan (HG) epitopes. (A) Immunolabeling of PTs of WT, etr3-ko, WTB and NR using LM20. (B) Fluorescence intensity was measured at PT apex. $n=80$ PTs for each line, error bars show SE, different letters show significant differences at $P=0.05$ (Dunn's test). The area delimited by a yellow dashed line (as shown on WTB) indicates the chosen zone for measuring the fluorescence mean intensity. (C) A representative picture of PTs cultured in the liquid growth medium for each line. (D) Measurement of PT length and diameter, in solid and liquid growth medium, $4 \mathrm{~h}$ after pollen grain imbibition, in the four tomato lines, $n=50$ PTs for each line. Error bars show SE and different letters show significant differences at $P=0.05$ (Tukey's HSD). Scale bars $=50 \mu \mathrm{m}$ in panels $\mathrm{A}$ and $\mathrm{C}$.

Figure 6. Effects of ETR mutations on the $\mathrm{Ca}^{2+}$ gradient in the apical regions of pollen tubes. (A, B, C, D) Representative images of $\mathrm{Ca}^{2+}$ gradient at maximal fluorescence over a 30 min growth period in WT, etr3-ko, WTB and NR respectively, scale bars $=10 \mu \mathrm{m}$. (E) Means of maximum fluorescence intensity measured in PT apex loaded with Fluo-4/AM over a 30 min growth period, $n=10$ PTs, error bars are SE, different small letters show significant differences at $P=0.05$ (Tukey's HSD). The zone delimited by a yellow dashed line indicates the region where the maximum fluorescence intensity has been measured. (F) Model showing that ethylene signaling regulates PT elongation through cell wall reshuffling by affecting activities of pectin methylesterases (PME) and through modifications in calcium gradient by affecting calcium channels (CNGC), calcium pumps (ACA) and $\mathrm{Ca}^{2+}$ effectors (CPK). Only enzyme families showing strong regulation in ETR LOF or GOF mutants are represented.

This article is protected by copyright. All rights reserved 
A

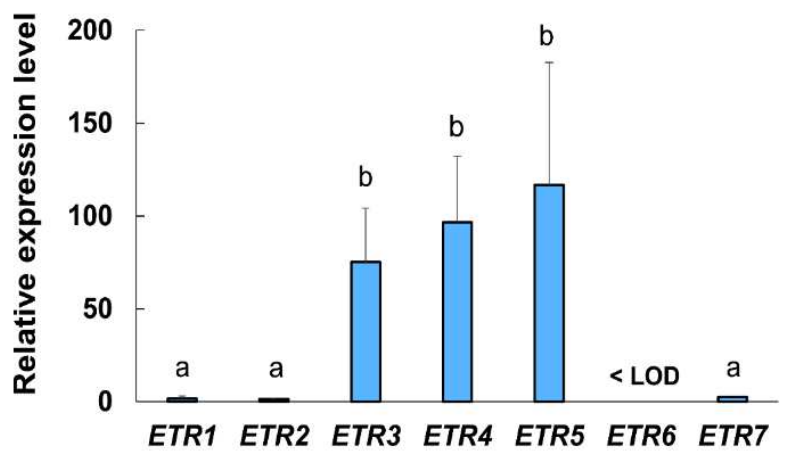

B
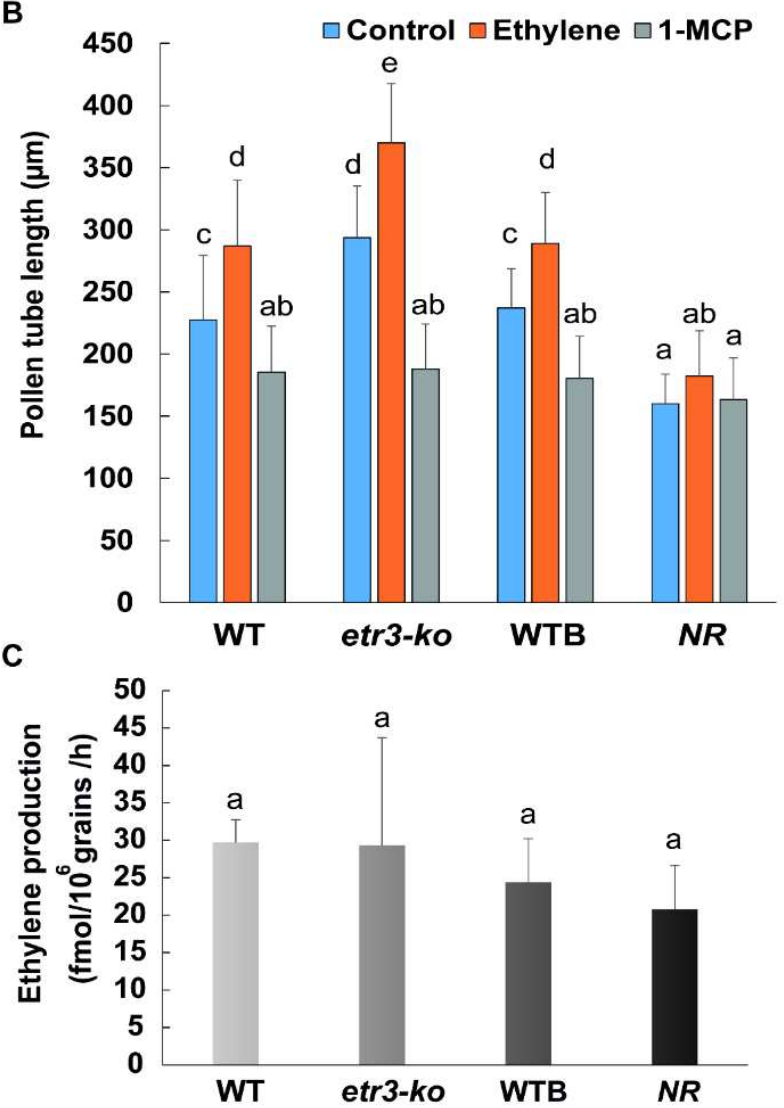

This article is protected by copyright. All rights reserved 
A

4

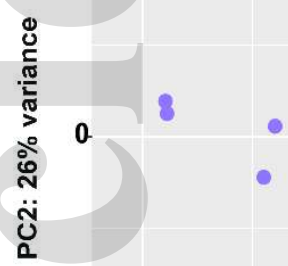

$\because$

$-5$

5

PC1: $42 \%$ variance

B NR vs WTB etr3-ko vs WT
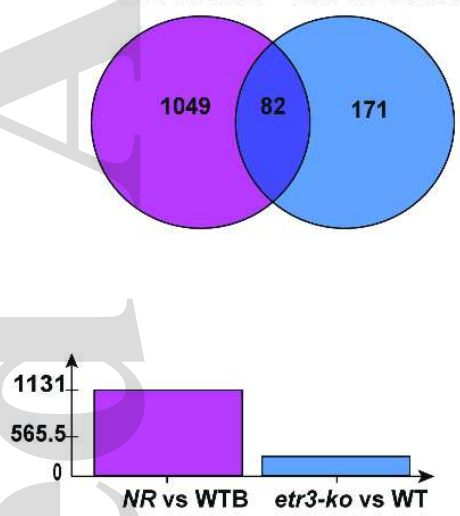

Up-regulated
C

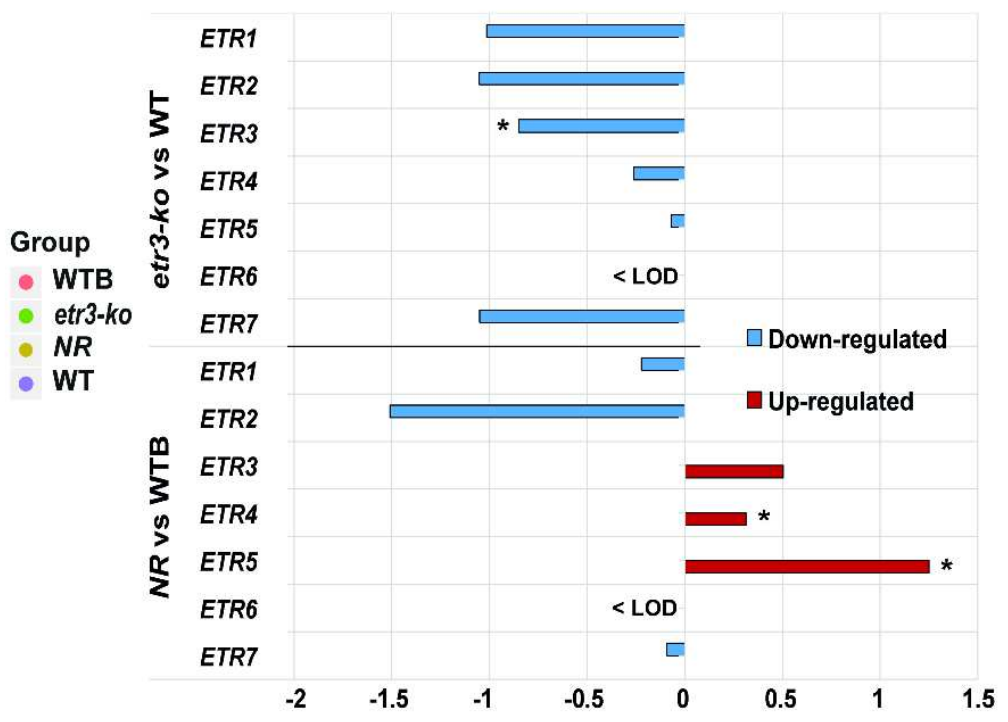

D
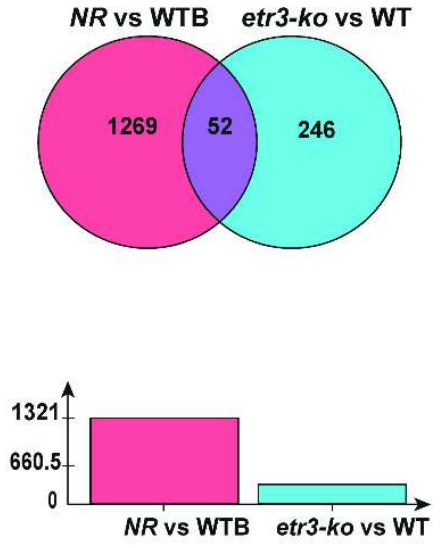

Down-regulated

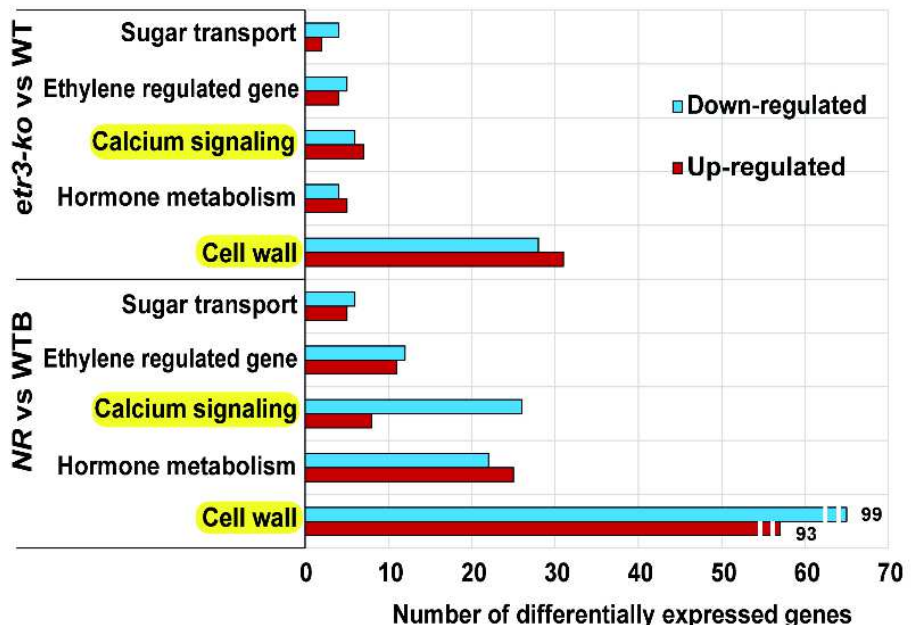

This article is protected by copyright. All rights reserved 
Solyc10g047570

Solyc09g075460

Solyc03g117750

Solyc03g116500

Solyc06g060170

Solyc03g007950

Solyc04g025440

Solyc03g113230

Solyc01g066420

Solyc01g066370

Solyc01g068120

Solyc05g054360

Solyc12g099410

PME Solyc12g099230

Solyc01g057220

Solyc05g052120

Solyc05g052110

Solyc02g075620

Solyc01g066410

Solyc04g010230

Solyc05g007080

PL Solyc02g031960

Solyc06g071020

Solyc01g010740

Solyc01g059940

PMEI Solyc01g088590

Solyc07g053330

Solyc07g062220

TBL Solyc10g009590

Solyc03g007170

PAC Solyc07g062560

PG Solyc03g116500

Solyc05g054360

Solyc07g017560

Solyc06g084620

Solyc01g068120

Solyc03g078260

PAE Solyc08g075030

Solyc03g058910

Solyc01g010740

Solyc03g113150

Solyc02g069300

Solyc01g059940

Solyc07g053330

$T B L$

Solyc03g006170

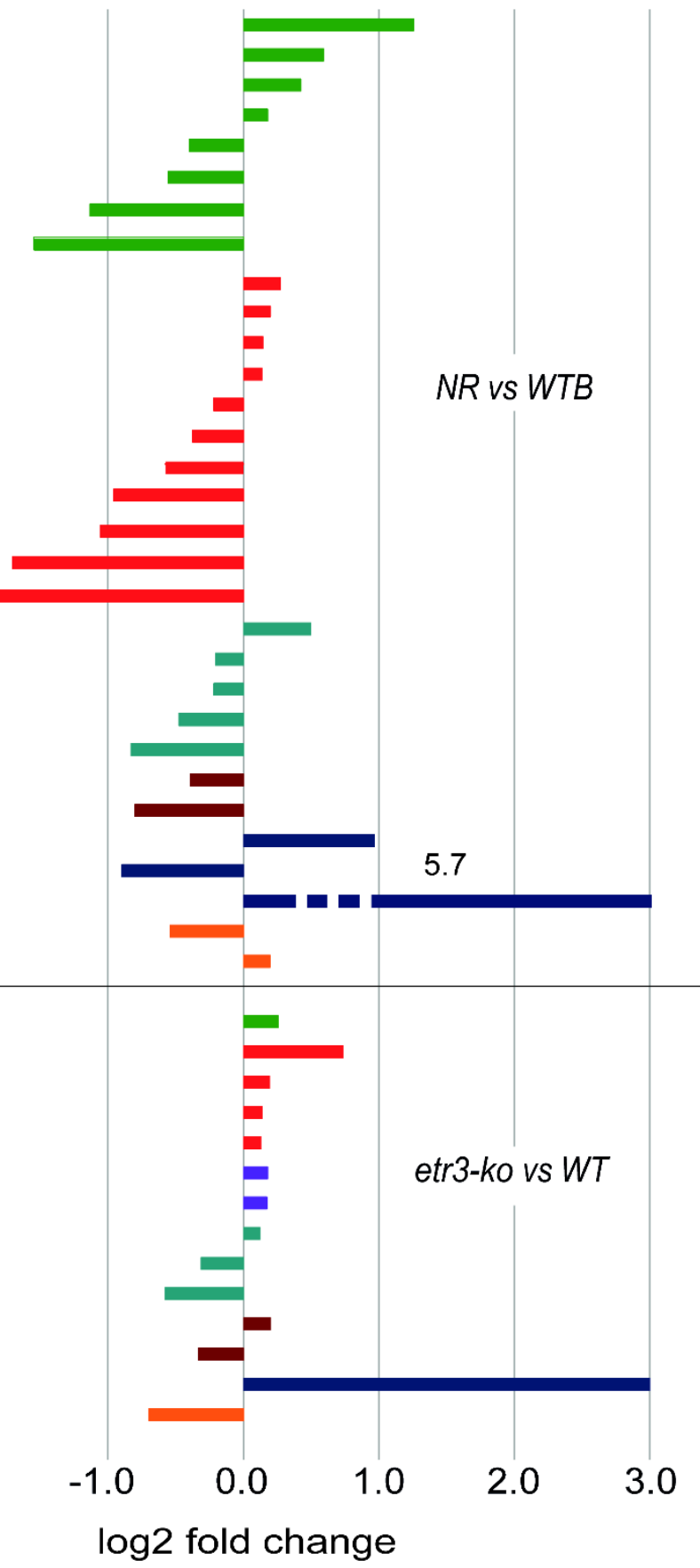

This article is protected by copyright. All rights reserved 
CIPKs

\section{CPKS}

Ca pumps

Calcium Channels

Calcium exchanger

CaMBP

CaBPs

CMLS

CBLS
CPKS

CaMBPs

CaBPs

CMLS
Solyc05g047600

Solyc03g006110

Solyc02g021440

Solyc12g099790

Solyc10g074570

Solyc03g123890

Solyc11g072880

Solyc03g114110

Solyc03g116850

Solyc12g088230

Solyc02g014560

Solyc07g056110

Solyc10g086060

Solyc09g009770

Solyc04g050050

Solyc05g054050

Solyc01g100380

Solyc03g118040

Solyc00g008680

Solyc12g089330

Solyc04g008270

Solyc08g021940

Solyc07g061950

Solyc03g080080

Solyc06g068700

Solyc01g094030

Solyc01g111520

Solyc06g083000

Solyc05g050750

Solyc02g067220

Solyc06g068960

Solyc01g010020

Solyc11g072270

Solyc02g063340

Solyc07g065820

Solyc11g064900

Solyc10g076900

Solyc10g086060

Solyc02g080400

Solyc09g009770

Solyc06g052040

Solyc00g008680

Solyc01g100380

Solyc04g055170

Solyc06g068960

Solyc10g079420

Solyc11g072240

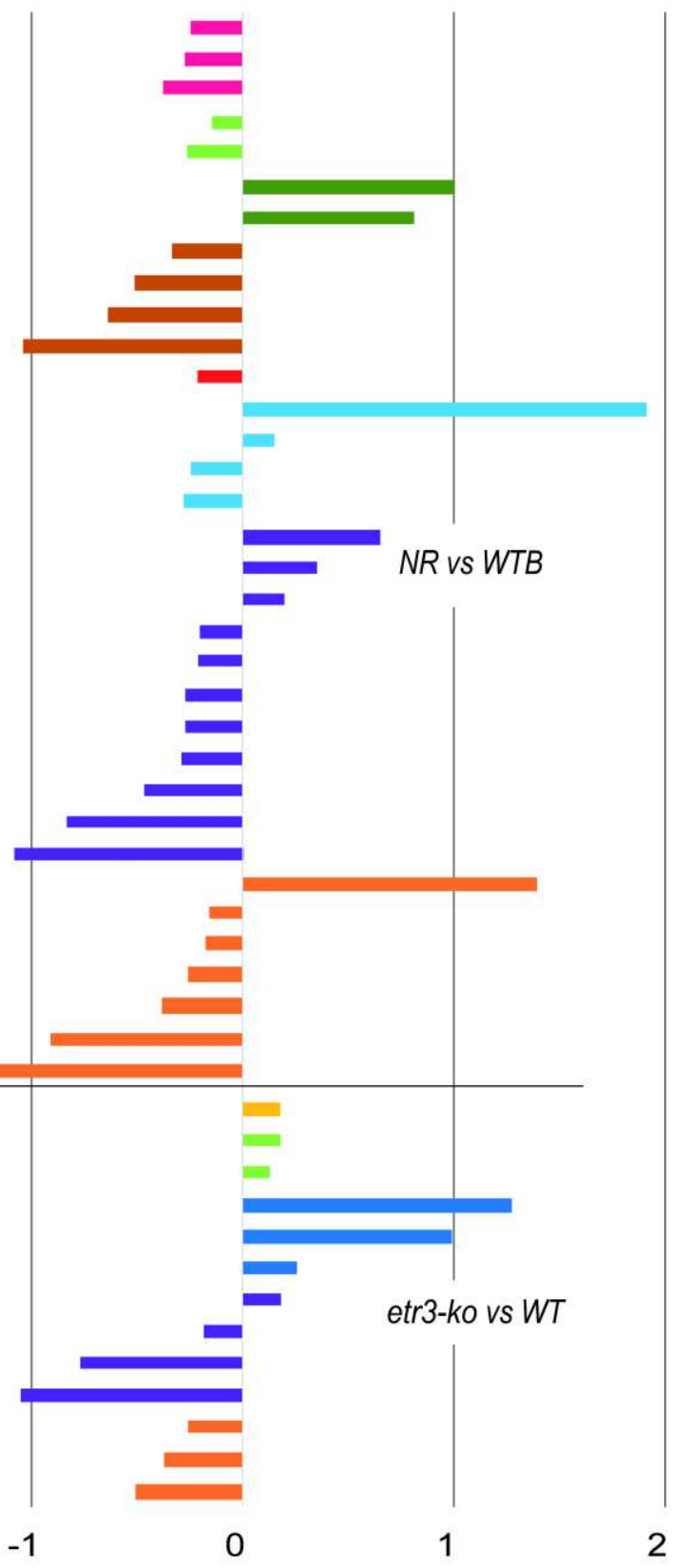

$\log 2$ fold change 
A WT

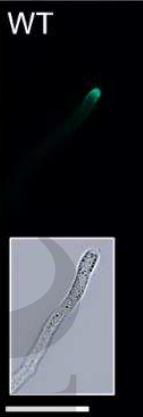

etr3-ko
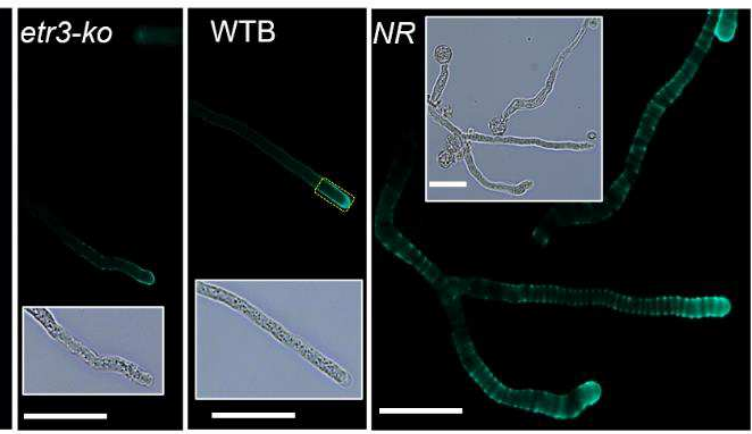

B

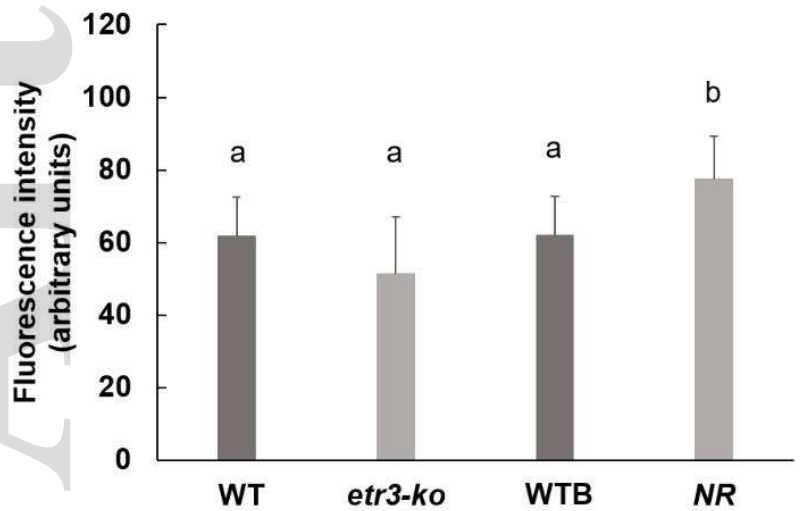

c
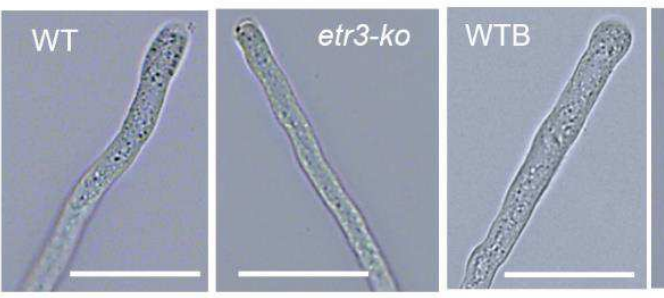

$N R$
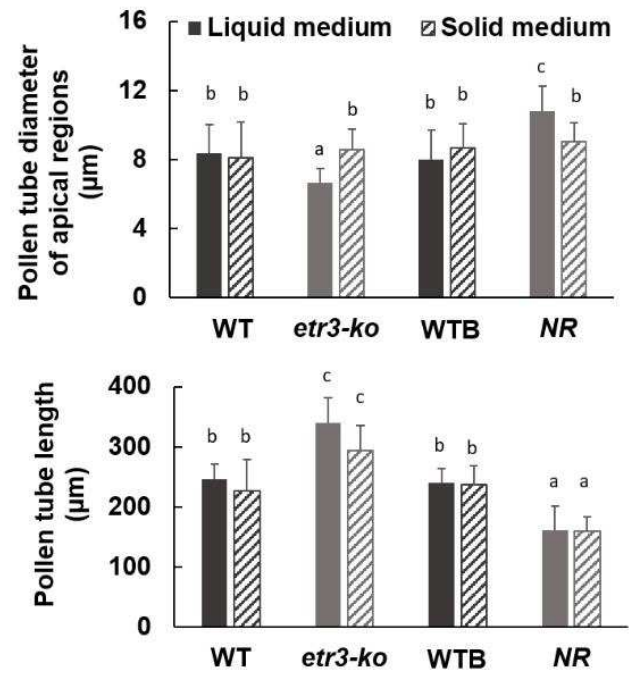

This article is protected by copyright. All rights reserved 


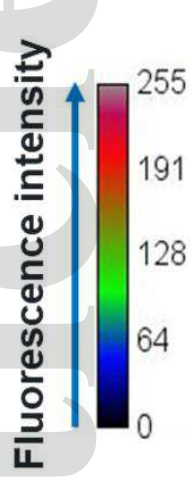

\section{A}
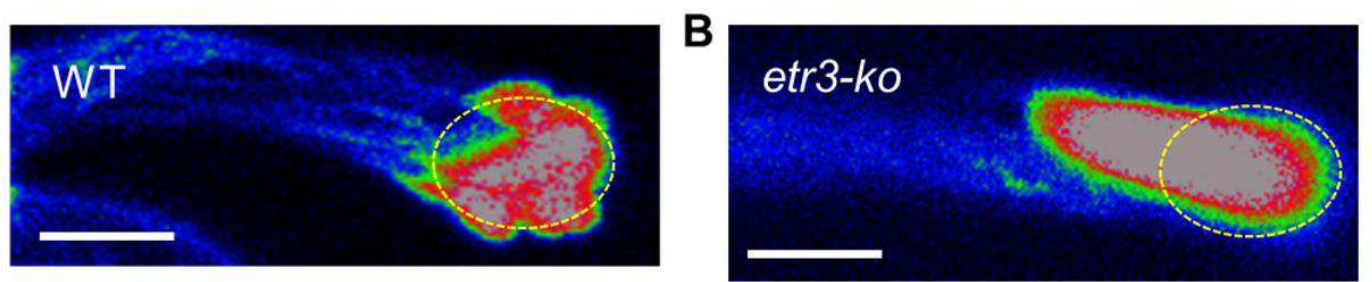

C
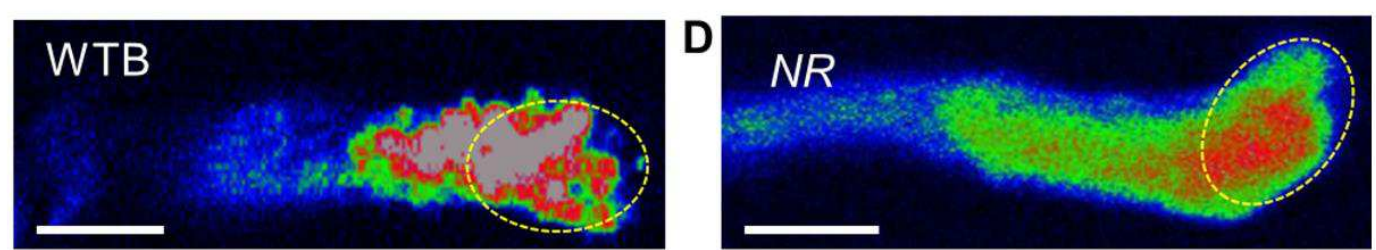

E
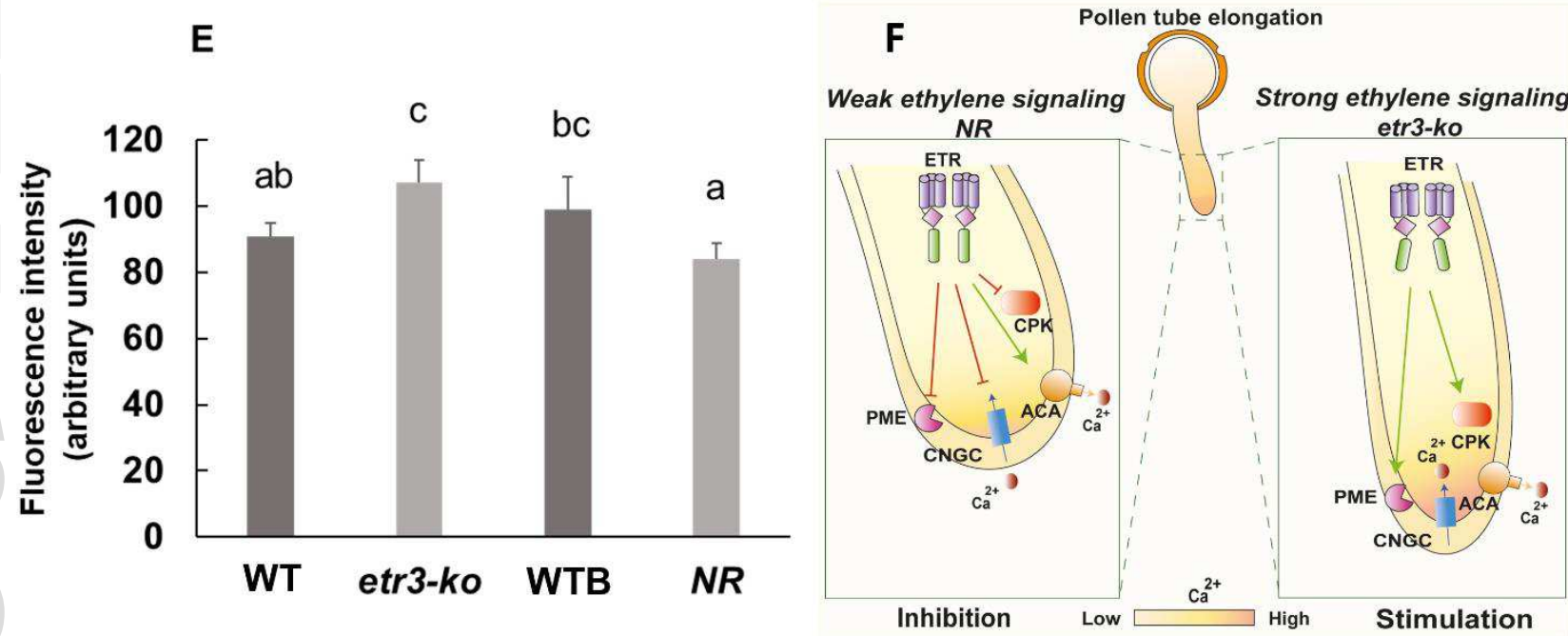

This article is protected by copyright. All rights reserved 